\title{
The lack of close binaries among hot horizontal branch stars in NGC 6752*
}

\author{
C. Moni Bidin ${ }^{1,2}$, S. Moehler ${ }^{3}$, G. Piotto ${ }^{1}$, A. Recio-Blanco ${ }^{1,4}$, Y. Momany ${ }^{1}$, and R. A. Méndez ${ }^{2}$ \\ 1 Dipartimento di Astronomia, Università di Padova, Vicolo dell'osservatorio 2, 35122 Padova, Italy \\ 2 Departamento de Astronomía, Universidad de Chile, Casilla 36-D, Santiago, Chile \\ e-mail: mbidin@das.uchile.cl \\ 3 Institut für Theoretische Physik und Astrophysik, Christan-Albrechts-Universität zu Kiel, 24098 Kiel, Germany \\ ${ }^{4}$ Observatoire de la Côte d'Azur, Dpt. Cassiopée, CNRS UMR 6202, BP 4229, 06304 Nice Cedex 04, France
}

Received 29 July 2005 / Accepted 5 January 2006

\section{ABSTRACT}

Aims. We present the results of a spectroscopic search for close binaries among horizontal branch (HB) stars in NGC 6752.

Methods. We used the ESO VLT-FORS2 instrument to obtain medium resolution $(R=4100)$ spectra of 51 hot $\mathrm{HB}$ stars with $8000 \mathrm{~K} \leq T_{\text {eff }} \leq$ $32000 \mathrm{~K}$ during four consecutive nights. Eighteen of our targets are extreme horizontal branch (EHB) stars with $T_{\text {eff }} \geq 22000 \mathrm{~K}$. Radial velocity variations were measured with cross-correlation techniques, previously evaluated the statistical and systematic errors associated with them.

Results. No close binary system has been detected among our 51 targets. The data corrected for instrumental effects indicate that the radial velocity variations are always below $\approx 15 \mathrm{~km} \mathrm{~s}^{-1}$ ( $3 \sigma$ level). From a statistical analysis of our results, we conclude that (at $95 \%$ confidence level) the fraction of binaries with a $\sim 0.5 M_{\odot}$ companion among EHB stars in NGC 6752 is smaller than $20 \%$.

Conclusions. This empirical evidence sharply contrasts with what has been found for hot subdwarfs in the field, and opens new questions about the formation of EHB stars in globular clusters (and possibly in the field as well).

Key words. stars: horizontal-branch - binaries: close - binaries : spectroscopic - globular clusters: individual: NGC 6752

\section{Introduction}

Although stellar evolution theory has successfully identified horizontal branch (HB) stars as post-helium flash stars of low (typically 0.7-0.9 $M_{\odot}$ in globular clusters) initial mass (Hoyle \& Schwarzschild 1955; Faulkner 1966), we still lack a comprehensive understanding of their nature. The large morphological differences (see for example Piotto et al. 2002) among HBs of Galactic globular clusters (GCs) are surely one of the most puzzling problems of stellar evolution. It is well known that cluster metallicity affects the HB morphology (Sandage \& Wallerstein 1960), but there are still many morphological differences among the HBs of GCs with the same metallicity (Sandage \& Wildey 1967; van den Bergh 1967), suggesting the presence of one or more second parameters. Many "second parameter" candidates have been proposed, but none has provided an overall explanation for all the available observations, and it is likely that the so-called "second parameter problem" is the consequence of a combination of parameters (Fusi Pecci et al. 1993).

\footnotetext{
* Based on observations with the ESO Very Large Telescope at Paranal Observatory, Chile (proposal ID 69.D-0682).
}

In more recent years, more and more GCs have been found to show an HB blue tail extending toward increasing effective temperatures and fainter visual magnitudes (Sosin et al. 1997; Rich et al. 1997; Ferraro et al. 1998; Piotto et al. 1999). This extreme horizontal branch (EHB) population with $T_{\text {eff }} \geq$ $20000 \mathrm{~K}$ is a challenging test of the theoretical models of the late evolutionary stages of low-mass stars, and their origin is still not understood (see below). The EHBs might also have important implications for extragalactic astronomy, since EHB stars have been identified as possibly being responsible for the UV upturn in elliptical galaxies. This UV upturn corresponds to the increase in flux with decreasing wavelength below $2500 \AA$ discovered in the bulge of M31 (Code 1969), and then found in almost all bright elliptical galaxies and many spiral bulges. It has been proposed that the EHB stars are the source of this increase in UV flux (see for example Greggio \& Renzini 1990, 1999; Brown et al. 2000). This idea has been reinforced by the discovery of EHB stars in metal-rich GCs like NGC 6388 and NGC 6441 (Rich et al. 1997) and in the Galactic bulge (Busso et al. 2005). As EHB stars evolve with time, the upturn is expected to change during the evolution of the galaxies, and to be almost absent for younger ellipticals (Greggio \& Renzini 1990; Tantalo et al. 1996). A knowledge of the 
formation mechanisms and evolution of EHB stars in different environments is therefore urgently needed in order to model the behavior of the UV upturn with age (redshift). The UV upturn could then also potentially be used as an age discriminator for elliptical galaxies.

While it is agreed that EHB stars are He-burning stars that have suffered heavy mass loss during their evolution (Iben \& Rood 1970; Faulkner 1972; D’Cruz et al. 1996), keeping only a thin external envelope with a mass around $0.02 M_{\odot}$ or lower, their actual formation mechanism remains unclear. Some mechanism able to enhance the mass loss must be responsible for their formation. As first proposed by Wilson \& Bowen (1984), many authors explored mass-loss mechanisms of the HB itself. Yong et al. (2000) successfully reproduce the morphology of blue HBs in metal-rich GCs with a constant mass-loss rate during $\mathrm{HB}$ evolution, but provide no explanation for the required rate. Vink \& Cassisi (2002) show that enhanced radiation-driven winds cannot account for the high mass-loss rates required, concluding that the most plausible mechanisms are unable to form EHB stars by mass loss on the HB. On the other hand, many authors propose heavy mass-loss rates during the previous red giant branch (RGB) phase (see for example Soker et al. 2001). Iben (1990) propose an alternative scenario, in which the EHB stars are formed from mergers of helium white-dwarf binary systems. Fusi Pecci et al. (1993) indicate that the GC density can enhance the presence of EHB stars, hinting that dynamical interactions may play an important role in their formation.

There is still a lack of models that can explain such heavy mass loss in the evolution of a single low-mass star. The binarity of EHB stars, as proposed by many authors (Mengel et al. 1976; Heber et al. 2002), might provide an explanation for their formation, since the dynamical interaction with a close compact companion can enhance the mass loss through a number of different binary-evolution channels (Han et al. 2002, see below), particularly during the RGB phase. The presence of a binary population in GCs is now well established (Hut et al. 1992; Bailyn 1993), and binaries can get closer and closer as a consequence of the dynamical interactions inside GCs (Heggie 1975). The idea that EHB stars are components of close binary systems has been strengthened by observations of field EHB stars, also known as subdwarf Btype stars (sdBs). Binaries have been found to be very common among them. Maxted et al. (2001) conclude from their observations that $69 \pm 9 \%$ of sdB stars are binaries. Morales-Rueda et al. (2003) recently measured the orbital periods $P$ and the semiamplitudes of radial velocity variation $K$ of 22 new binary sdBs, increasing the number of sdBs for which these quantities are known to 38 . Thirty of them have periods below 3 days (22 with $P \leq 1$ day), showing that they are almost all very close systems, with $K$ usually exceeding $50 \mathrm{~km} \mathrm{~s}^{-1}$ (only 7 exceptions) and easily greater than $100 \mathrm{~km} \mathrm{~s}^{-1}$. A more recent search for binaries among field sdB stars (Napiwotzki et al. 2004) led to a much lower $(42 \%)$ close binary fraction than expected from previous results. Napiwotzki et al. (2004) point out that their sample contained a much higher percentage of faint, i.e. distant, stars than the one of Maxted et al. (2001). The Napiwotzki et al. sample may therefore extend to greater distances from the
Galactic plane and thus be contaminated by thick-disk or halo members. This could imply that a relation of binary frequency with metallicity and/or age is present.

Han et al. (2003) find from binary population synthesis techniques that $76-89 \%$ of the sdBs should be close binaries. Han et al. (2002) analyzed in detail the main binary evolution channels that can lead to the sdB formation. Comparing their models with the available empirical data, they find that a very efficient common-envelope (CE) channel can fit the observed distribution of close-binary $\mathrm{sdB}$ periods, though it is not possible to fit the observed cumulative luminosity functions, as shown by Lisker et al. (2005), showing that there are still some problems with the binary scenario. In the CE channel, the progenitor of the sdB star is a giant that fills its Roche lobe near the tip of the red giant branch and experiences mass transfer during which the core of the giant and the companion (a normal main-sequence star or a white dwarf) spiral towards each other inside a common envelope formed out of the giant envelope. Once enough orbital energy has been released, the envelope is ejected and the system becomes a short-period binary.

There are alternative mechanisms suggested for the formation of the EHB stars. Recent observational results on $\omega$ Centauri (Bedin et al. 2004; Piotto et al. 2005), M3-M 13 (Caloi \& D'Antona 2005), and NGC 2808 (D'Antona et al. 2005) have given new impulse to the idea that EHB stars may result from a second generation of stars enriched in helium by pollution from intermediate-mass AGB star ejecta (D'Antona et al. 2002; Lee et al. 2005).

In the attempt to shed some light on the origin of EHB stars in globular clusters, we want to test in this paper the possibility that they are stars that have experienced greatly enhanced mass loss during the evolution in a close binary system. We started our spectroscopic search for close binaries among the EHB stars of NGC 6752. This GC is an ideal target as it is dynamically evolved, shows an extended and well-populated blue HB (as shown in Fig. 1), a large population of X-ray sources in the core that could be cataclysmic variables (Pooley et al. 2002), and it is supposed to have a large main-sequence binary population (Rubenstein \& Bailyn 1997).

In a forthcoming paper, we will present and discuss the main atmospheric parameters ( $T_{\text {eff }}, \log g, \log \frac{n_{\mathrm{He}}}{n_{\mathrm{H}}}$, mass $)$ derived from a complementary set of spectra obtained during the same observational run. In the following, we concentrate on the radial velocity and radial velocity variation measurements, and on their implication for the close binary scenario in the formation of EHB stars.

\section{Observation and data reduction}

The spectra were acquired during four nights of observation, from June 11 to June 14, 2002, at the VLT-UT4 telescope equipped with the FORS2 spectrograph in MXU mode. Fiftyone HB stars were selected from the photometric catalog of NGC 6752 by Momany et al. (2002). The stars are well distributed along the HB from the cool edge $\left(T_{\mathrm{eff}} \approx 8000 \mathrm{~K}\right)$ to the $\mathrm{EHB}\left(T_{\mathrm{eff}} \approx 30000 \mathrm{~K}\right)$, and were divided into 3 fields for multi-object spectroscopy. The position of our targets on the 


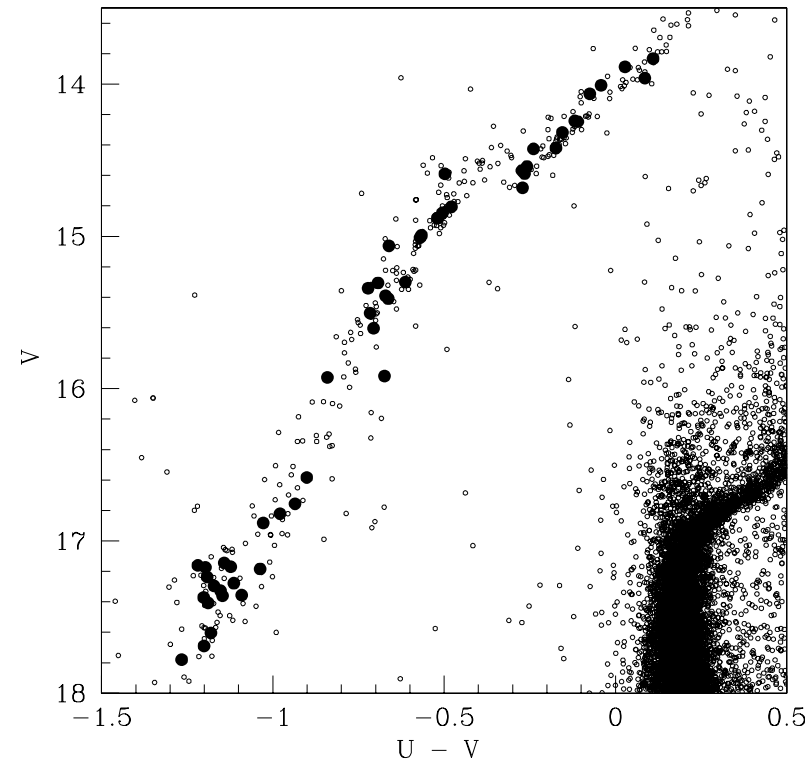

Fig. 1. Positions of target stars (solid dots) in the color-magnitude diagram of NGC 6752. Data from Momany et al. (2002).

HB is indicated in Fig. 1 and their radial distribution is shown in Fig. 2.

The list of targets, their coordinates, $V$ magnitudes, and the relevant parameters extracted from the color-magnitude diagram and our spectra are given in Table 1. The approximate effective temperatures were obtained from the $U-V$ colors by Momany et al. (2002), by applying the color-temperature relation derived from the temperatures spectroscopically measured by Moehler et al. (2000) on a sample of HB stars in the same cluster as in Fig. 3. Combining the dispersion of the points around the fitted line in Fig. 3, and the photometric errors in $U-V$ we estimated the error in effective temperature to be approximately $5 \%$.

During each night, up to two pairs of $1800 \mathrm{~s}$ exposures were secured in each field with grism $1400 \mathrm{~V}+18$, as shown in Table 2. The slit width was 0.'5 and the resulting resolution $1.2 \AA$. The exposures were always collected in pairs and subsequently summed (see Sect. 3.1) except on the 3rd night for field A, when only one $1800 \mathrm{~s}$ exposure was acquired. Just before each pair of exposures, a slit image (without grism) was taken, and these frames were used in the correction of instrumental effects that affected the data (Sect. 3.2). The bias, flat, and lamp images were acquired before and at the end of each observing night. The observed spectral range was $\approx 1300 \AA$ wide but, due to the different positions of the slits within the masks, the central wavelengths of the spectra varied. The bluer spectra reached $\approx 4200 \AA$ in the blue edge encompassing the $\mathrm{H}_{\gamma}$ line and the $4471 \AA$ HeI doublet, while others extended on the red side up to $\approx 6000 \AA$. The $\mathrm{H}_{\beta}$ line was always inside the observed spectral range, except for star 28695 . This cool target was excluded from our analysis due to the lack of strong lines in the spectral range.

Data reduction from the multi-spectrum frames to the onedimensional calibrated spectra was performed with standard

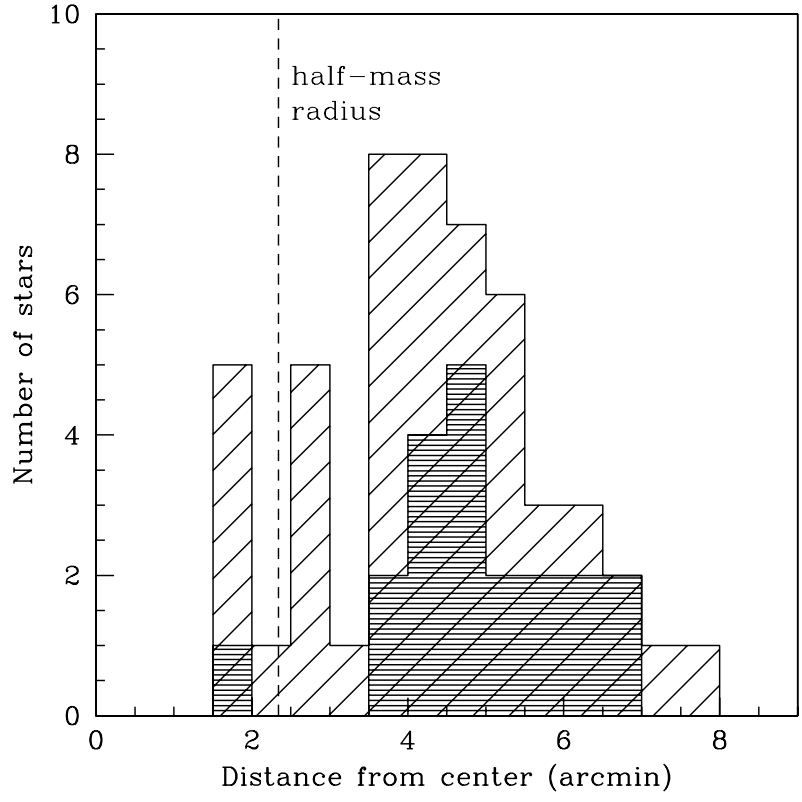

Fig. 2. Radial distribution of the observed stars. The dark shaded area indicates hot stars $\left(T_{\text {eff }} \geq 20000 \mathrm{~K}\right)$. The half-mass radius from Harris (1996) is also indicated.

MIDAS $^{1}$ procedures. All slitlets were extracted from the full frames (bias, wavelength calibration, flat field, science spectra) and reduced independently. The wavelength calibration (wlc) was performed with the $\mathrm{HeNeHgCd}$ lamp images, fitting a 3 rd order polynomial to the dispersion relation. We re-binned the $2 \mathrm{D}$ frames to constant wavelength steps of $0.25 \AA /$ pix. The extraction region for target and sky varied strongly from star to star because different parameters affected the selection, mainly the crowding level and the object magnitude. We tried to extract all the spectra with an optimal extraction routine (Horne 1986), but for some stars (mainly the brightest ones) the procedure gave bad results, so in these cases we opted for a simple average over the extraction region.

No flux calibration was applied to the spectra, because they were continuum-normalized for the measurement of radialvelocity variations.

\section{Measures}

\subsection{RV-variation measurement}

Radial velocities (RVs) were measured with the crosscorrelation (CC) technique (Tonry \& Davis 1979), using the fxcor IRAF $^{2}$ task. Before co-adding each pair of spectra we measured the RV variation between them, in order to verify significant variation had occurred and that no

${ }^{1}$ ESO-MIDAS is the acronym for the European Southern Observatory Munich Image Data Analysis System that is developed and maintained by the European Southern Observatory (http://www . eso.org/projects/esomidas/).

${ }^{2}$ IRAF is distributed by the National Optical Astronomy Observatories, which are operated by the Association of Universities for Research in Astronomy, Inc., under cooperative agreement with the National Science Foundation. 
Table 1. List of the observed stars with their physical parameters. IDs, coordinates (RA and Dec), magnitudes $V$ and color $(U-V)$ are from Momany et al. (2002). The absolute heliocentric radial velocities $\left(V_{\text {rad }}\right)$ were measured in the present work, the effective temperatures were obtained from the $U-V$ color of the targets, with the relation $(U-V)$ vs. $T_{\text {eff }}$ shown in Fig. 3 and described in the text. The estimated error for temperatures is $\sigma_{T}=5 \%$.

\begin{tabular}{|c|c|c|c|c|c|c|c|c|}
\hline Field & Slit & ID & $\begin{array}{l}\text { RA (J2000) } \\
\text { hh:mm:ss }\end{array}$ & $\begin{array}{c}\operatorname{Dec}(\mathrm{J} 2000) \\
\text { ': ':" }\end{array}$ & V & $\begin{array}{r}V_{\mathrm{rad}} \\
\mathrm{km} \mathrm{s}^{-1}\end{array}$ & $\begin{array}{r}T_{\text {eff }} \\
\mathrm{K}\end{array}$ & $(U-V)$ \\
\hline $\mathrm{A}$ & 1 & 14770 & $19: 11: 32.563$ & $-59: 59: 37.63$ & 17.247 & $-35 \pm 10$ & 30300 & -1.192 \\
\hline A & 2 & 11634 & $19: 11: 16.084$ & $-60: 00: 27.33$ & 14.431 & $-43 \pm 10$ & 10000 & -0.174 \\
\hline A & 3 & 14944 & $19: 11: 22.485$ & $-59: 59: 35.51$ & 15.401 & $-44 \pm 10$ & 15600 & -0.671 \\
\hline A & 4 & 15026 & 19:11:12.319 & $-59: 59: 34.51$ & 14.018 & $-36 \pm 9$ & 9100 & -0.042 \\
\hline A & 5 & 16551 & $19: 11: 14.253$ & $-59: 59: 13.90$ & 15.420 & $-44 \pm 10$ & 15500 & -0.663 \\
\hline A & 6 & 15395 & $19: 11: 05.940$ & $-59: 59: 29.51$ & 17.337 & $-40 \pm 8$ & 29000 & -1.153 \\
\hline A & 7 & 20919 & $19: 11: 10.496$ & $-59: 58: 15.25$ & 13.844 & $-34 \pm 7$ & 8100 & +0.110 \\
\hline A & 8 & 18782 & $19: 11: 16.428$ & $-59: 57: 45.90$ & 14.601 & $-35 \pm 10$ & 13100 & -0.498 \\
\hline A & 9 & 17941 & $19: 11: 04.827$ & $-59: 58: 02.02$ & 15.928 & $-39 \pm 8$ & 16700 & -0.674 \\
\hline A & 10 & 20302 & $19: 11: 16.912$ & $-59: 57: 13.20$ & 16.594 & $-36 \pm 7$ & 20800 & -0.901 \\
\hline A & 11 & 26756 & $19: 11: 25.759$ & $-59: 56: 17.07$ & 14.599 & $-21 \pm 9$ & 10800 & -0.265 \\
\hline A & 12 & 27181 & $19: 11: 24.177$ & $-59: 56: 04.10$ & 15.073 & $-34 \pm 10$ & 15400 & -0.661 \\
\hline A & 13 & 24849 & 19:10:59.399 & $-59: 57: 05.99$ & 14.578 & $-49 \pm 9$ & 10800 & -0.273 \\
\hline A & 14 & 27604 & $19: 11: 17.021$ & $-59: 55: 50.56$ & 15.937 & $-36 \pm 9$ & 19200 & -0.841 \\
\hline A & 15 & 28231 & $19: 11: 18.560$ & $-59: 55: 27.51$ & 17.367 & $-29 \pm 6$ & 26900 & -1.091 \\
\hline A & 16 & 26760 & 19:10:59.788 & $-59: 56: 17.51$ & 15.515 & $-37 \pm 7$ & 16400 & -0.716 \\
\hline A & 17 & 28554 & $19: 11: 07.939$ & $-59: 55: 14.16$ & 17.289 & $-19 \pm 8$ & 27600 & -1.114 \\
\hline A & 18 & 28693 & 19:11:08.108 & $-59: 55: 08.53$ & 17.306 & $-26 \pm 10$ & 29600 & -1.172 \\
\hline A & 19 & 28947 & $19: 11: 04.724$ & $-59: 54: 56.61$ & 16.832 & $-36 \pm 8$ & 23200 & -0.979 \\
\hline B & 1 & 4964 & $19: 10: 49.893$ & $-60: 04: 08.04$ & 14.552 & $-47 \pm 8$ & 10700 & -0.258 \\
\hline B & 2 & 49317 & $19: 11: 15.879$ & $-60: 06: 00.17$ & 13.889 & $-35 \pm 8$ & 8600 & +0.028 \\
\hline B & 3 & 5455 & $19: 10: 52.734$ & $-60: 03: 36.84$ & 17.371 & $-34 \pm 6$ & 28700 & -1.147 \\
\hline B & 4 & 5487 & $19: 10: 57.554$ & $-60: 03: 35.02$ & 16.766 & $-43 \pm 10$ & 21800 & -0.935 \\
\hline B & 5 & 5134 & $19: 11: 09.624$ & $-60: 03: 56.23$ & 15.615 & $-40 \pm 8$ & 16200 & -0.706 \\
\hline B & 6 & 4672 & $19: 11: 21.548$ & $-60: 04: 31.46$ & 17.181 & $-33 \pm 8$ & 27900 & -1.123 \\
\hline B & 7 & 5201 & $19: 11: 26.748$ & $-60: 03: 51.85$ & 17.615 & $-37 \pm 7$ & 29900 & -1.181 \\
\hline B & 8 & 5865 & $19: 11: 21.577$ & $-60: 03: 14.90$ & 17.195 & $-44 \pm 9$ & 25100 & -1.037 \\
\hline B & 9 & 7843 & $19: 1$ & $-60: 021: 55.92$ & 15.317 & $-41 \pm 8$ & 16000 & -0.693 \\
\hline B & 10 & 6284 & $19: 11: 29.780$ & $-60: 02: 54.90$ & 17.155 & $-36 \pm 6$ & 28600 & -1.142 \\
\hline B & 11 & 10257 & 19:11:07.698 & $-60: 00: 53.00$ & 14.075 & $-37 \pm 10$ & 9300 & -0.075 \\
\hline B & 12 & 10625 & $19: 11: 21.006$ & $-60: 00: 45.37$ & 17.701 & $-24 \pm 9$ & 30700 & -1.201 \\
\hline B & 13 & & & $-60: 01: 30.83$ & 17.790 & $-33 \pm 9$ & 33500 & -1.266 \\
\hline B & 14 & 10711 & $19: 11: 28.425$ & $-60: 00: 43.55$ & 17.418 & $-31 \pm 7$ & 30200 & -1.189 \\
\hline $\mathrm{C}$ & 1 & 11609 & $19: 10: 36.021$ & $-60: 00: 28.10$ & 15.311 & $-37 \pm 9$ & 14600 & -0.613 \\
\hline $\mathrm{C}$ & 2 & 14664 & $19: 10: 41.367$ & $-59: 59: 39.90$ & 13.972 & $-7 \pm 9$ & 8300 & +0.086 \\
\hline $\mathrm{C}$ & 3 & 14727 & $19: 10: 39.624$ & $-59: 59: 39.13$ & 14.253 & $-33 \pm 9$ & 9600 & -0.119 \\
\hline $\mathrm{C}$ & 4 & 35186 & $19: 10: 13.293$ & $-60: 00: 03.25$ & 14.443 & $-34 \pm 9$ & 10600 & -0.239 \\
\hline $\mathrm{C}$ & 5 & 35662 & 19:10:22.119 & $-59: 59: 30.30$ & 15.010 & $-40 \pm 9$ & 14000 & -0.566 \\
\hline $\mathrm{C}$ & 6 & 35499 & 19:10:10.920 & $-59: 59: 41.57$ & 14.895 & $-35 \pm 9$ & 13400 & -0.518 \\
\hline $\mathrm{C}$ & 7 & 36242 & $19: 10: 23.020$ & $-59: 58: 47.17$ & 15.024 & $-31 \pm 7$ & 14100 & -0.570 \\
\hline $\mathrm{C}$ & 8 & 36480 & $19: 10: 23.013$ & $-59: 58: 30.37$ & 16.899 & $-35 \pm 8$ & 24800 & -1.028 \\
\hline $\mathrm{C}$ & 9 & 36502 & $19: 10: 18.896$ & $-59: 58: 28.74$ & 14.863 & $-35 \pm 9$ & 13200 & -0.505 \\
\hline $\mathrm{C}$ & 10 & 36830 & 19:10:02.476 & $-59: 58: 03.50$ & 17.388 & $-33 \pm 8$ & 30700 & -1.202 \\
\hline $\mathrm{C}$ & 11 & 38095 & $19: 10: 26.616$ & $-59: 56: 23.29$ & 15.358 & $-35 \pm 9$ & 16100 & -0.700 \\
\hline $\mathrm{C}$ & 12 & 38087 & $19: 10: 18.120$ & $-59: 56: 23.89$ & 17.190 & $-37 \pm 8$ & 30500 & -1.197 \\
\hline $\mathrm{C}$ & 13 & 32470 & $19: 10: 22.172$ & $-59: 55: 54.36$ & 14.681 & $-27 \pm 8$ & 10800 & -0.271 \\
\hline $\mathrm{C}$ & 14 & 28695 & $19: 10: 35.134$ & $-59: 55: 08.70$ & 14.329 & - & 9900 & -0.155 \\
\hline $\mathrm{C}$ & 15 & 38504 & 19:10:15.308 & $-59: 55: 41.62$ & 14.823 & $-39 \pm 9$ & 12900 & -0.155 \\
\hline $\mathrm{C}$ & 16 & 39008 & $19: 10: 25.034$ & $-59: 54: 41.75$ & 17.178 & $-15 \pm 11$ & 31400 & -1.219 \\
\hline $\mathrm{C}$ & 17 & 38889 & $19: 10: 09.836$ & $-59: 54: 56.10$ & 14.897 & $-48 \pm 9$ & 13400 & -0.520 \\
\hline $\mathrm{C}$ & 18 & 38963 & $19: 10: 03.450$ & $-59: 54: 46.56$ & 14.264 & $-17 \pm 10$ & 9600 & -0.110 \\
\hline
\end{tabular}




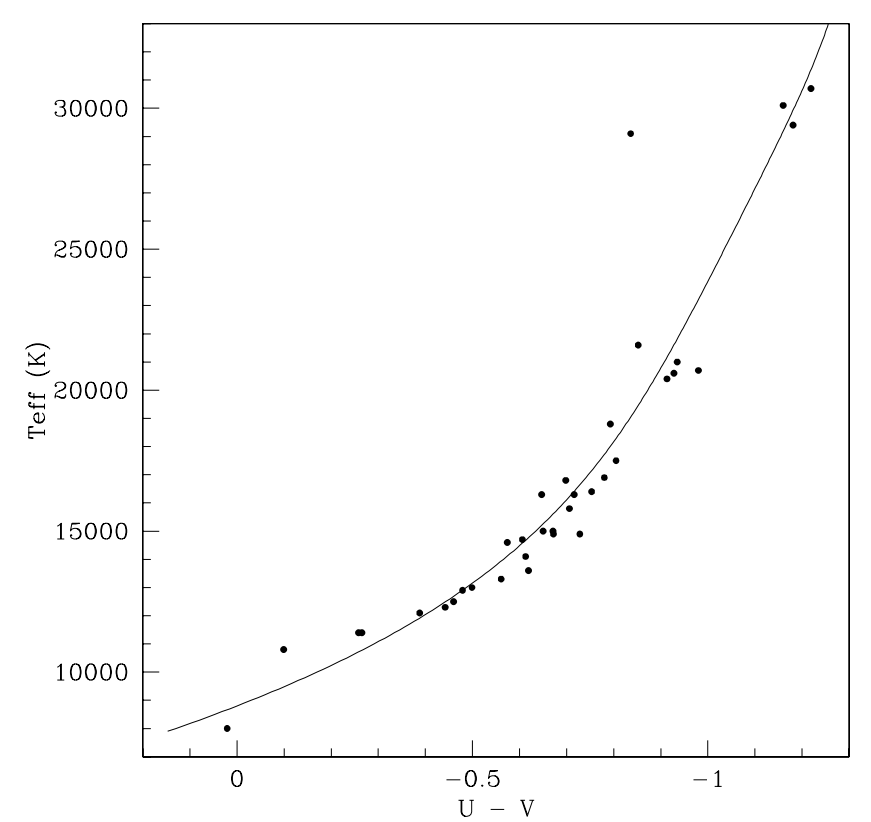

Fig. 3. Plot used to derive the approximate effective temperatures of our targets. The points are the effective temperatures measured by Moehler et al. (2000) using models with solar metallicity, plotted against the $U-V$ color in the Momany et al. (2002) photometry. Seven stars listed in Moehler et al. (2000) have been excluded since their identification in the Momany et al. (2002) photometry is dubious. The line indicates the $T_{\text {eff }}$ vs. color relation obtained by a fit of the data points, used to estimate the temperatures of the stars observed in our work. The point at $U-V \approx-0.8, T_{\text {eff }} \approx 29000 \mathrm{~K}$ were excluded from the fit.

information was lost due to averaging. The summed spectra will be referred to with the field and the night of observation (from 12 to 15), with an additional letter ( $a$ or b) when two pairs were acquired in the same night and field. The A14 spectra come from a single $1800 \mathrm{~s}$ exposure, since only one frame was acquired that night, and in our analysis we took their lower $\mathrm{S} / \mathrm{N}$ into account.

For each target star all the spectra were cross-correlated, thus performing $10 \mathrm{CCs}$ for each star in fields $\mathrm{A}$ and $\mathrm{B}$ and 6 in field $\mathrm{C}$, covering different temporal intervals from one hour to 3.1 days. In each measurement the first spectrum (in temporal order) was assumed as template (the choice of which of the two spectra is used as template affects only the sign of the $\mathrm{RV}$ variation).

For each RV measurement the cross-correlation function (CCF) was computed and the position of its peak determined with a Gaussian fit (see for example Recio-Blanco et al. 2004; Dubath et al. 1990, for a description of the procedure). In the measurement concerning Balmer lines, the CCFs have Gaussian shapes only in the peak but not in the wings, and sometimes they are asymmetric; whereas the CCFs obtained from weak lines are approximated well by a Gaussian, but they are often distorted due to low $\mathrm{S} / \mathrm{N}$. Therefore the fit was restricted to the central portion around the peak, but different fits were tried, varying the width of the CCF region fitted, in order to find the best estimate for the RV variations.

Our analysis focused on the $\mathrm{H}_{\beta}$ line, and 432 measurements were performed in the 4830-4890 $\AA$ spectral range $\left(\mathrm{H}_{\beta}\right.$ line
Table 2. List of $1800 \mathrm{~s}$ exposures acquired each night. The UT of the start of the exposures (hour and minutes) is indicated, and also the averaged seeing (variations between the exposures in the same night are within 0 !' 1$)$.

\begin{tabular}{c|cccc}
\hline \hline Field & \multicolumn{4}{|c}{ Night } \\
& 12 & 13 & 14 & 15 \\
\hline \multirow{3}{*}{ A } & \multicolumn{5}{|c}{$2: 44$} & & \\
& $8: 43$ & $3: 15$ & $9: 34$ & $7: 47$ \\
& $9: 14$ & $3: 56$ & $8: 18$ & \\
& & $4: 27$ & & \\
\hline \multirow{3}{*}{ B } & $5: 55$ & & & \\
& $6: 27$ & $5: 08$ & $8: 33$ & $8: 58$ \\
& $6: 59$ & $5: 39$ & $8: 59$ & $9: 29$ \\
& $7: 30$ & & & \\
\hline \multirow{3}{*}{ C } & & $6: 39$ & & \\
& - & $8: 04$ & $7: 05$ & $6: 36$ \\
& - & $8: 34$ & $7: 33$ & $7: 06$ \\
& & & $7: 59$ & \\
\hline Average seeing & 0.9 & $1{ }^{\prime \prime} 2$ & 1.3 & $11^{\prime \prime} 4$ \\
\hline
\end{tabular}

with full wings). We also compared these results with measurements from other parts of the spectrum, both to clarify the influence of noise on the measured RV variations and to overcome the intrinsic weakness of the $\mathrm{CC}$ technique for blended lines in double spectra (see for example Zucker \& Mazeh 1994). Therefore, although our survey was intended to be based on analysis of the $\mathrm{H}_{\beta}$, all the measurements were repeated, cross-correlating the entire spectra (with and without $\mathrm{H}_{\beta}$ ) and $\mathrm{H}_{\gamma}$ (alone and with $\mathrm{H}_{\beta}$ ) when it fell inside the spectral range, for a total number of $1532 \mathrm{CCs}$. The presence of many metallic lines in the spectra of stars with $11500 \leq T_{\text {eff }} \leq 18000 \mathrm{~K}$ (see Fig. 4), due to radiative levitation of heavy elements (Glaspey et al. 1989; Behr 2003), gave good CCFs even without the $\mathrm{H}$ lines. On the other hand, the low $\mathrm{S} / \mathrm{N}$ and the lack of useful lines in the spectra of hot stars $\left(T_{\text {eff }} \geq 18000 \mathrm{~K}\right)$ usually prevented cross-correlating the entire spectra without $\mathrm{H}_{\beta}$, and then only spectral sections with the strongest helium lines were cross-correlated in place of these measurements, with quite uncertain results (see Sect. 4.1). Fourier filters of various shapes (Brault \& White 1971) were applied to almost all the noisy spectra, obtaining better CCFs but unchanged results for the most part.

The [OI] $5577 \AA$ A sky line was used as zero-point in order to correct possible spectral shifts due to differences between lamp and star spectra. The sky line fell outside the spectral range for 13 stars, preventing us from correcting the spectra before the measurements. We found that the differences in the sky line position in the spectra between each pair of frames is a linear function of $Y$-position (perpendicular to the dispersion direction) of the slits in the masks (Fig. 5). From the measured sky line positions, we then calculated these differences for each pair of spectra, and applied them as corrections to the RV variations measured with the CCs. For the 13 stars missing the [OI] line, we calculated the corrections from their 


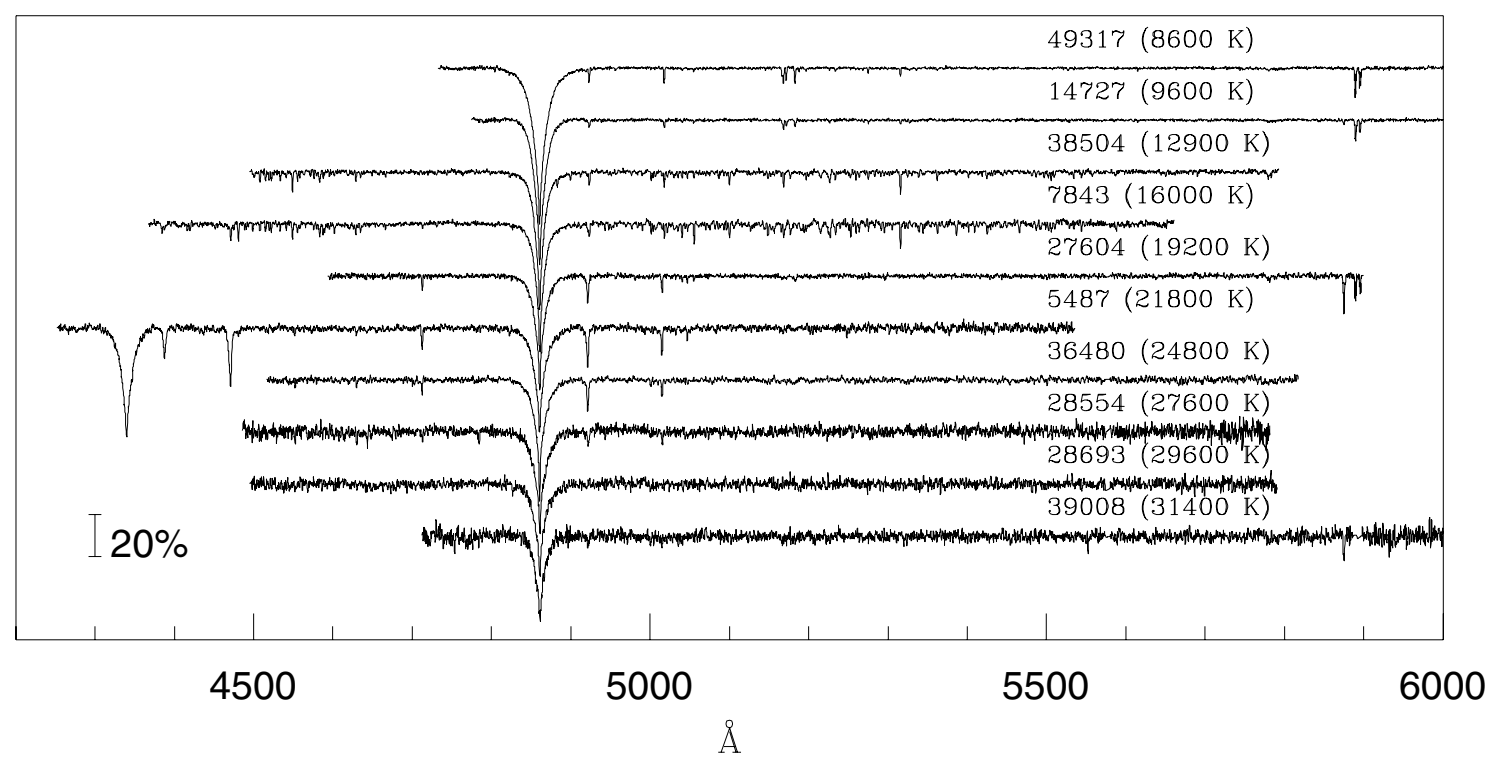

Fig. 4. A sample of the collected spectra, normalized and ordered by increasing $T_{\text {eff }}$. The increasing noise with temperature is clearly visible, as well as the large quantity of metallic lines for stars with $11500 \leq T_{\text {eff }} \leq 18000$.

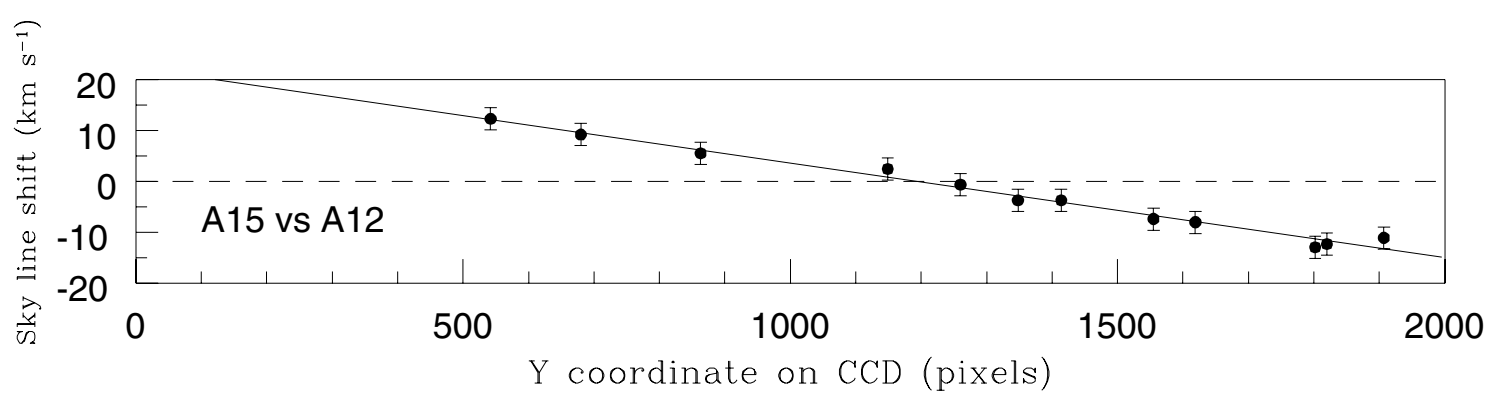

Fig. 5. An example of the corrections to re-position the 5577 A sky line to its laboratory wavelength. The differences (in $\mathrm{km} \mathrm{s}^{-1}$ ) in the position of the sky line in the spectra A15 and A12 are plotted as a function of the $Y$-positions of the slits on the CCD (in pixels). The corrections for the few stars without the sky line in the observed spectral range hwere obtained from their $Y$-position by the least-square solution (straight line in the figure). The error bar in the plot is the $1 \sigma$ estimated for this corrections (see Sect. 4.4).

$Y$-coordinates by means of the least-square fit obtained by using the spectra with the $[\mathrm{OI}]$ line.

\subsection{Correction of the RV measures}

After the skyline-based correction mentioned above, we were forced to correct the measured RV variations again, since they showed a clear correlation with the displacement (along the dispersion direction) of the stars with respect to the center of the slit. The effect resulted in an evident systematic error, up to $10-12 \mathrm{~km} \mathrm{~s}^{-1}$, of the same order of magnitude for all the stars within the same pair of frames (usually with a dependence on $Y$-position on CCD, probably due to rotation of the mask and/or the field). In the slit frames we measured the position of the stars relative to the center of the slits with a Gaussian fit of the stellar profile parallel to the dispersion direction. Then, we obtained the differences in these positions between pairs of frames, and translated them from pixels to $\mathrm{km} \mathrm{s}^{-1}$ with the instrumental relation $38.2 \mathrm{~km} \mathrm{~s}^{-1}$ pixel $^{-1}$, in order to evaluate the effect of this shift on the RV measurements. Actually, it is not the real stellar profile that has been fitted, but the star profile convolved with the narrow slit, that generates the spectrum on the CCD. In fact, for narrow slits, the relation between the movement of the star behind them and the induced RV variation should be flatter than the dispersion relation, but a shift of the center of the profile that reaches the grism through the slit is expected to induce an RV variation given by the dispersion relation, with good approximation. This is confirmed by the plot in Fig. 6, where we compared the two quantities (shift of the profile and RV variation) for each star and each pair of frames: the points show a good agreement with the expected relation indicated by the straight line. Although the plot confirms that it is a good solution for relating the two quantities, it also possibly indicates that it is just a first-order approximation, because a deviation from the straight line for higher displacements can be seen. The real solution could be an S-shaped function. Nevertheless we adopted a linear approximation, that still gave good enough corrections for our purposes and our overall errors, in order to correct the RV variations with quantities that depend only on the measured displacements and not on the $\mathrm{RV}$ variations themselves (as would be the case if, for example, we fit the points in Fig. 6 to obtain a higher-order relation).

We then compared the displacements of the profiles and the $\mathrm{RV}$ variations for each star, as a function of the $Y$ coordinate on CCD. A typical example is shown in Fig. 7 (upper panel). 


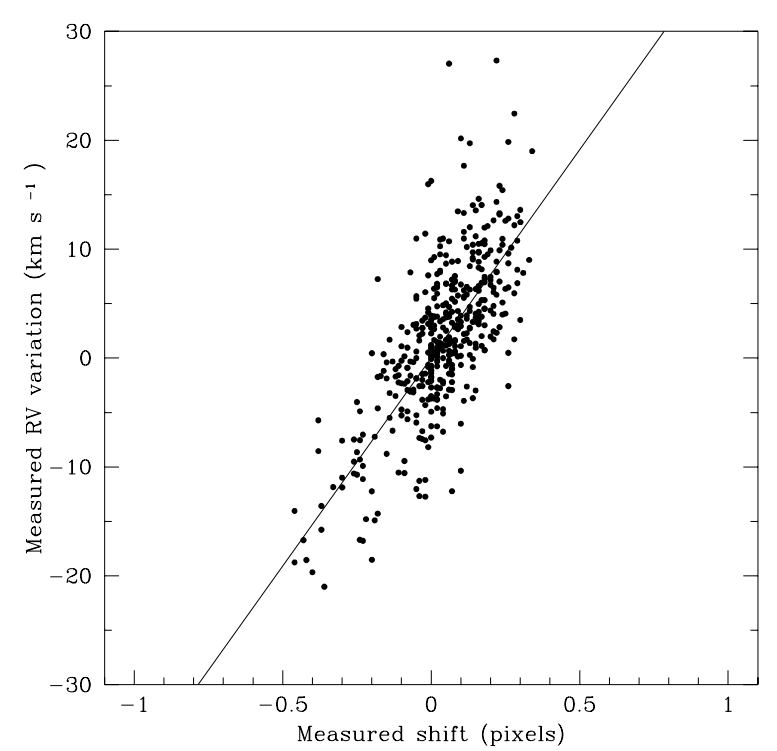

Fig. 6. Displacements of stars with respect to the center of the slit plotted against the RV variations measured from CCs. The straight line indicates the instrumental relation 1 pixel $=38.2 \mathrm{~km} \mathrm{~s}^{-1}$ given by the dispersion of the spectra.

A dependence on slits' $Y$-position can also be seen, but with a certain scatter due to the random errors that affected the stellar fitting procedure in the narrow slits. We preferred to derive the final corrections by applying the value of the least-square fit of these plots to each star instead of the real shifts measured starby-star, in order to avoid introducing additional noise into the data.

For frames B12b and A13a, the slit image was not acquired immediately before the exposures, but one hour before for frame $\mathrm{B} 12 \mathrm{~b}$ and one hour after for A13a. In both cases, the corrections do not agree with the RV variations observed, but a constant shift between them is clear (Fig. 7, middle panel) and has of the same amount for both frames. This indicates a time-dependent instrumental movement (probably a shift of the mask in its housing) that shifted the spectra slightly $\left(\approx 7 \mathrm{~km} \mathrm{~s}^{-1}=0.2\right.$ pixels $)$. Either way, the slit image for frames B12a and B12b was the same (collected just before the first, B12a), and they were taken in sequence, without any telescope-pointing change, and calibrated with the same lamp image. For all these reasons, it is reasonable to assume that the observed shift of the sky line on the CCD between the frames B12a and B12b was only due to this time-dependent instrumental movement. Therefore we think that we have been able to measure and correct this effect properly.

The corrections derived for frame B13 do not overlap the RV observed variations (Fig. 7, lower panel). Since it is the only frame out of 14 for which this happens, its slit frame seems not to be reliable for some reason. Probably the mask moved inside its frame between the slit image acquisition and the spectra exposure. We estimated a mask shift of $0.13-0.34$ pixels with a rotation 0.00928 degrees. This is not unlikely and similar effects have been noted by other observers before. Since we could not both find an explanation and prove it, we preferred to exclude this frame from further analysis. The uncorrected

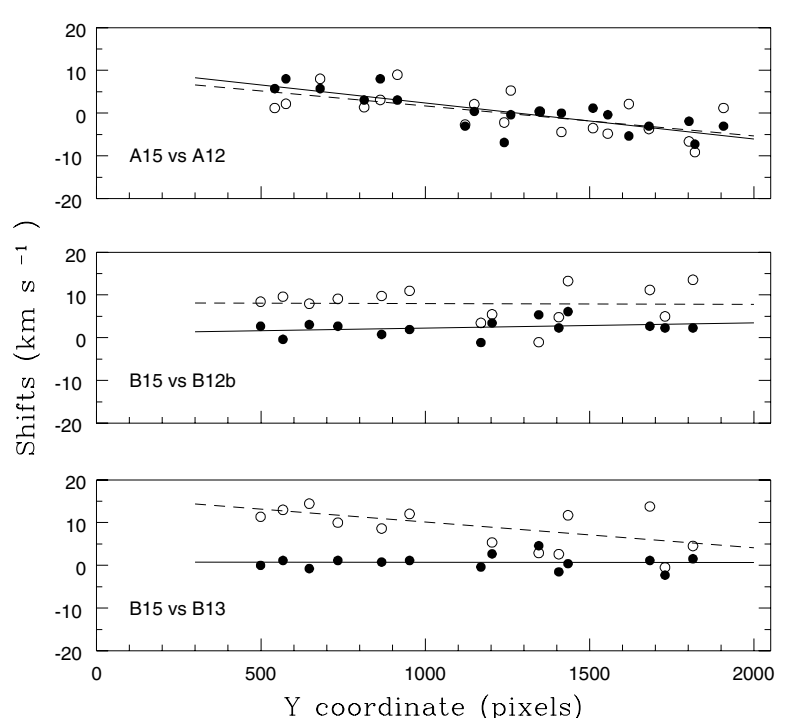

Fig. 7. Comparison between the measured displacement of stars inside the slit (solid points), translated from pixels to $\mathrm{km} \mathrm{s}^{-1}$, and the RV observed variations (open points). The lines are the least-square solutions for the two sets of data. Upper panel: a typical plot (frames A15 and A12), in which the two sets of data are very similar. Central panel: same plot involving frame B12b. A shift between the two lines is evident. Bottom panel: same plot involving frame B13. The two sets of data do not agree.

RV variations observed are still useful and give some information (see Sect. 5), although only in a qualitative way.

\subsection{Absolute RV measures}

We measured absolute RVs in order to check the cluster membership of the observed stars by means of CCs with the template star HD 188112, a binary sdB star with known ephemeris (Heber et al. 2003). The results are shown in Col. 7 of Table 1. The final absolute RVs have been corrected for sky line position, as already described in Sect. 3.2. All the stars show an absolute RV in agreement with that of the cluster $\left(-27.9 \mathrm{~km} \mathrm{~s}^{-1}\right.$, Harris 1996) within $2 \sigma$, and can thus be considered RV cluster members.

\section{Error analysis}

We performed an accurate error analysis in order to estimate the significance of the measured RV variations. We analyzed all the main error sources, and estimated a standard error $\sigma$ for each of them. The final errors associated to the results presented in Table 4 were obtained as their quadratic sum.

\subsection{Cross-correlation errors}

The error in the $\mathrm{CC}$ procedure is obtained by the $\mathrm{CC}$ theory as $\sigma_{\mathrm{CC}}=\frac{N}{8 B(1+r)}$

where

$r=\frac{h}{\sqrt{2} \sigma_{\mathrm{a}}}$ 


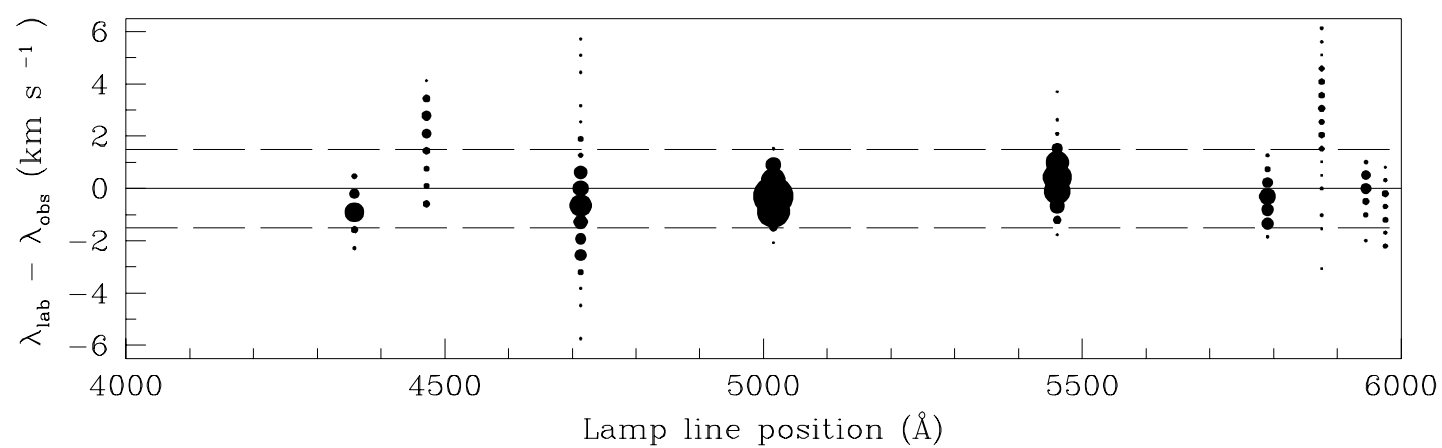

Fig. 8. Shift of lamp lines compared to their laboratory wavelength, in $\mathrm{km} \mathrm{s}^{-1}$. Each circle is proportional to the frequency of the datum. The dashed lines indicate the $1 \sigma$ dispersion.

and $N$ is the number of bins, $B$ the highest wavelength in which the CCF Fourier transform has an appreciable value, $h$ is the height of the CCF peak, and $\sigma_{\mathrm{a}}$ the rms of the antisymmetric part of the CCF (see Tonry \& Davis 1979, for details). This error is computed directly by the fxcor IRAF task during each $\mathrm{CC}$ procedure. In the measurements with Balmer lines it is usually between $0.5 \mathrm{~km} \mathrm{~s}^{-1}$ (for high $\mathrm{S} / \mathrm{N}$ spectra) and $2 \mathrm{~km} \mathrm{~s}^{-1}$ (for asymmetric CCFs), but occasionally CCFs with bad profiles lead to a $\mathrm{CC}$ error up to $5 \mathrm{~km} \mathrm{~s}^{-1}$. The measurements without Balmer lines are affected by much higher errors for hot stars (usually 3-5 $\mathrm{km} \mathrm{s}^{-1}$ ), whereas for cooler stars the accuracy remained quite unchanged due to the numerous metallic lines in their spectra.

\subsection{Wavelength calibration errors}

The precision and reliability of the wlc procedure, which is essential for our analysis, was tested in each of the 186 lamp images used. These images were calibrated with the coefficients obtained in the wlc procedure, and the position of 9 bright lamp lines were measured with a Gaussian fit and compared to their laboratory wavelength. This analysis pointed out the level of inaccuracy in the wlc procedures that could affect the RV measures. The obtained data are plotted in Fig. 8.

No systematic error was found. Only the lines 4471 and $5875 \AA$ seem calibrated redder than the theoretical wavelength, but every night the mean shift of the former was not greater than the wlc error, and the latter line lies in the extreme red part of the spectra, never used in the CCs, and between two well-calibrated lamp lines. The dispersion of the data is in the range $1.3-1.7 \mathrm{~km} \mathrm{~s}^{-1}$, slightly but not significantly varying among the nights and the three fields. They were considered as an estimate of the wlc error $\sigma_{\text {wlc }}$, which has been counted twice (in quadrature) in the final error estimate because in each $\mathrm{CC}$ two spectra are involved.

\subsection{Extraction and fit errors}

Two additional sources of error are present in the measurement procedure. The first one is related to the extraction of spectra from the 2D images, as the choice of the aperture width can change the profile of spectral lines (mostly in noisy spectra or in the presence of cosmic spikes) slightly, and the $\mathrm{CC}$ procedure
Table 3. Standard deviations $\left(\mathrm{km} \mathrm{s}^{-1}\right)$ of the differences between the $\mathrm{RV}$ measurements obtained with different fits and spectral extractions In field B the bluest stars have been distinguished from the others, and "single spectrum" indicates the measurements involving the notsummed spectra A14.

\begin{tabular}{cccc}
\hline \hline$\sigma_{\text {ex }}$ & \multicolumn{3}{c}{ Field } \\
& A & B & C \\
\hline cool stars & 1.49 & 0.73 & 1.18 \\
hot stars & 2.48 & 2.11 & 2.69 \\
very hot stars & - & 2.66 & - \\
single-spectr. cool stars & 2.10 & - & - \\
single-spectr. hot stars & 3.46 & - & - \\
\hline
\end{tabular}

is sensitive enough to reveal the differences. The second, more important, is related to the choice of the number of CCF points used to compute its Gaussian fit. For distorted CCFs, this error is much greater than $\sigma_{\mathrm{CC}}$, since the final result could depend on the chosen fit.

These error sources were evaluated together by reextracting all the spectra in different manners and performing new measures with different fits in the $\mathrm{H}_{\beta}$ wavelength. We repeated $75 \%$ of the measurements (326 out of 432), chosen in a stochastic way in order to avoid selection effects in this choice. The distribution of the differences among these new measurements and the ones used for this paper give the combined extraction and fit error $\sigma_{\text {ex }}$ (Table 3). We divided the spectra in groups with similar $\mathrm{S} / \mathrm{N}$, i.e. hot and cool stars $\left(T_{\text {eff }} \lessgtr 20000 \mathrm{~K}\right)$ and very hot stars in field $B\left(T_{\text {eff }} \approx 30000 \mathrm{~K}\right)$. In field $\mathrm{A}$, the extraction and fit errors for the CCs involving the not-summed A14 spectra (much noisier) were evaluated separately and, as expected, they came out much greater than the others.

\subsection{Sky line position errors}

The correction procedure described in Sect. 3.1 introduced an additional error, due to the uncertainty in the measurement of the $5577 \AA$ sky line position. We assumed this error to be equal to the dispersion of the corrections around the leastsquare solutions when plotted against the slit $Y$-position, as in Fig. 5. We estimated $\sigma_{\text {sky }}=0.025 \AA$, as expected for a fit 
error $\sigma=0.1$ pixels. A similar value was obtained by analyzing the Gaussian fits of the sky lines. Then for the measurements in $\mathrm{H}_{\beta}$ we have

$\sigma_{\text {sky }}=0.025 \AA=1.54 \mathrm{~km} \mathrm{~s}^{-1}$

to be considered twice (with proper error propagation), as each correction involves two independent measurements.

\subsection{Correction to RV measures}

The corrections to the RV measurements described in Sect. 3.2 have been an additional source of error. For each pair of frames, we obtained the corrections by plotting the differences in stellar positions as in Fig. 7. The standard deviations of residuals with respect to the least-square solution were calculated for each pair, and used as the estimated error $\sigma_{\text {corr }}$ introduced by this procedure. It changed only slightly from one pair to the other, being always $0.7 \leq \sigma_{\text {corr }} \leq 1.4 \mathrm{~km} \mathrm{~s}^{-1}$.

\section{Results}

\section{1. $R V$ variations with $H_{\beta}$}

We obtained the most accurate and reliable RVs using the $\mathrm{H}_{\beta}$ line. The $\mathrm{RV}$ variations from all the possible combinations of the observed spectra are listed in Table 4. For each star, Fig. 9 shows the absolute value of the measured maximum $\mathrm{RV}$ variation.

All the variations are small, usually below $10 \mathrm{~km} \mathrm{~s}^{-1}$, and never further than $3 \sigma$ from zero; therefore no $R V$ variation can be considered significant. The variations are only slightly larger in the blue part of the HB, but with larger errors due to decreasing $\mathrm{S} / \mathrm{N}$ in the spectra.

The highest variation plotted in Fig. $9\left(21.7 \mathrm{~km} \mathrm{~s}^{-1}\right)$ refers to star 28231. We consider this datum interesting but particularly dubious, since it was measured in frame A14 where we measured higher variations, due to higher noise of the spectra, and star 28231 showed particularly distorted line profiles. This variation is not confirmed by the measurements in other wavelengths, and this star shows no other remarkable variation on the other nights. Therefore, it is highly probable that the relatively high $\mathrm{RV}$ variation is only due to noise-distorted $\mathrm{H}_{\beta}$ wing profile.

Table 4 also gives the results concerning the frame B13 (uncorrected for the star position inside the slit, see Sect. 3.2), although these have been excluded from our analysis and from the plot in Fig. 9. It can be seen that this exclusion represents no substantial loss of information, since the RVs appear scattered around a certain systematic value different from zero, with no larger RV variations than the typical $3 \sigma$ interval. Then even when including the data from the uncorrected frame B13, we can confirm our general results.

\section{2. $R V$ variations in other wavelengths}

The results with $\mathrm{H}_{\gamma}$, when available in the observed spectral range, always confirm the ones using $\mathrm{H}_{\beta}$. Occasionally the difference reaches $\approx 10 \mathrm{~km} \mathrm{~s}^{-1}$ (Fig. 10, central panel), but this agrees with a Gaussian distribution of the differences with a dispersion equal to our estimated errors.

The measurements with weak metallic lines gave very good results for cool stars (Fig. 10, upper and bottom panel), because of the large number of lines and the good $\mathrm{S} / \mathrm{N}$ of the spectra. The RV variations are always small, and the general trend confirms the results we had using $\mathrm{H}_{\beta}$ (the differences are on average below $3 \mathrm{~km} \mathrm{~s}^{-1}$ ). For hot stars the results obtained using weak lines (mainly He lines) are not very reliable for many reasons: they are not uniform, since the number of useful lines changes from spectrum to spectrum; the wavelength is not clearly determined, so the applied corrections introduce some uncertainty, due to the translations from $\AA$ to velocity quantities; the lines are few and weak, and then easily distorted by noise spikes. Still all the RV variations are within the $3 \sigma$ error bars, although the errors are larger than in the previously discussed measurements. For some stars the maximum RV variations occasionally reach $30 \mathrm{~km} \mathrm{~s}^{-1}$ (Fig. 10, bottom panel). It is worth noting that these differences always tend toward limiting high variations in $\mathrm{H}_{\beta}$, never toward emphasizing them, and this is also clear from the fact that, in spite of these great differences, the maximum $\mathrm{RV}$ variations are on absolute value that is the same order of magnitude as in $\mathrm{H}_{\beta}$ or just slightly higher. For example, both the stars 28231 and 28947, which showed the highest $\mathrm{RV}$ variations in $\mathrm{H}_{\beta}$, show no great variation in the weak line measurements. Therefore the measurements with weak lines confirm the results with $\mathrm{H}_{\beta}$, and indicate that the higher $\mathrm{RV}$ variations observed with $\mathrm{H}_{\beta}$ are simply due to random errors.

\subsection{Binary detection probability in our observations}

In order to better understand the significance of our results we calculated the probability $d$ of detecting a binary in our observations as a function of the period $P$. In order to relate the period and the maximum semiamplitude of the RV curve, we assumed a circular orbit and a mass of $0.5 M_{\odot}$ for both components. These assumptions are representative of the typical binary systems observed in field sdB stars.

For each value of $P$ we considered 50 possible values of $v \sin (i)$ (where $i$ is the inclination of the orbit along the line of sight), and 50 possible values of the phase $T_{0}$ (equally distributed at constant step in the range $0 \leq T_{0} \leq P$ and $0 \leq$ $v \sin (i) \leq 1)$. Then, we calculated how many of these $2500 \mathrm{bi}$ nary systems would have been detected in our observations, defining as "detection" an RV variation of more than $20 \mathrm{~km} \mathrm{~s}^{-1}$ (our $3 \sigma$ for hot stars) between any two of our observed epochs. Finally we weighted the probabilities for the three fields by the number of hot stars observed in each, in order to derive the average detection probability $d$ of our observations.

The results are shown in Fig. 11. The probability of detecting a binary with periods $P<5$ days is usually higher than $80 \%$, and reaches $90 \%$ for periods shorter than one day. There is an evident loss of sensitivity around $P=1$ day, since this is the typical temporal interval between two observing epochs. 
Table 4. RV variations for target stars.

\begin{tabular}{|c|c|c|c|c|c|c|c|c|c|c|}
\hline star & $13 \mathrm{a}$ vs. 12 & $13 \mathrm{~b}$ vs. 12 & $13 \mathrm{~b}$ vs. $13 \mathrm{a}$ & 14 vs. 12 & 14 vs. $13 \mathrm{a}$ & 14 vs. $13 b$ & 15 vs. 12 & 15 vs. $13 \mathrm{a}$ & 15 vs. $13 b$ & 15 vs. 14 \\
\hline $1 \mathrm{a}$ & $-5.5 \pm 4.7$ & $-10.0 \pm 4.5$ & $-2.0 \pm 4.2$ & $-3.1 \pm 5.2$ & $-2.5 \pm 4.9$ & $-1.1 \pm 5.0$ & $-4.0 \pm 5.5$ & $0.0 \pm 4.3$ & $3.3 \pm 4.9$ & $3.6 \pm 5.0$ \\
\hline $2 \mathrm{a}$ & $-3.2 \pm 4.2$ & $-3.3 \pm 4.0$ & $-0.3 \pm 3.9$ & $-4.2 \pm 4.4$ & $-0.9 \pm 3.9$ & $-2.4 \pm 4.2$ & $-3.0 \pm 4.0$ & $0.4 \pm 3.9$ & $0.6 \pm 3.7$ & $1.0 \pm 3.9$ \\
\hline $3 a$ & $0.0 \pm 4.2$ & $1.5 \pm 4.1$ & $2.2 \pm 3.8$ & $-1.6 \pm 4.4$ & $-1.1 \pm 3.9$ & $-2.4 \pm 4.2$ & $2.4 \pm 4.1$ & $2.5 \pm 3.8$ & $0.6 \pm 3.7$ & $0.7 \pm 3.9$ \\
\hline $4 a$ & $-0.4 \pm 4.2$ & $-2.2 \pm 4.1$ & $-1.8 \pm 3.7$ & $-1.0 \pm 4.4$ & $-0.6 \pm 3.9$ & $-0.8 \pm 4.1$ & $-2.0 \pm 4.0$ & $-1.6 \pm 3.8$ & $0.0 \pm 3.7$ & $-1.0 \pm 3.9$ \\
\hline $5 \mathrm{a}$ & $1.9 \pm 4.3$ & $-1.4 \pm 4.3$ & $-3.2 \pm 3.9$ & $2.7 \pm 4.4$ & $0.9 \pm 3.9$ & $2.1 \pm 4.1$ & $-0.3 \pm 4.0$ & $-2.3 \pm 3.9$ & $1.2 \pm 3.7$ & $-3.1 \pm 3.9$ \\
\hline $6 a$ & $-2.3 \pm 4.7$ & $-2.5 \pm 4.5$ & $0.4 \pm 4.3$ & $5.7 \pm 7.3$ & $9.0 \pm 5.9$ & $2.3 \pm 7.2$ & $4.7 \pm 4.6$ & $7.5 \pm 4.3$ & $6.6 \pm 4.6$ & $-2.2 \pm 7.6$ \\
\hline $7 \mathrm{a}$ & $0.2 \pm 4.2$ & $-1.7 \pm 4.1$ & $-2.2 \pm 3.8$ & $-0.4 \pm 4.4$ & $-0.7 \pm 4.0$ & $-0.5 \pm 4.0$ & $-3.2 \pm 4.0$ & $-3.4 \pm 3.8$ & $-1.2 \pm 3.7$ & $-2.7 \pm 3.8$ \\
\hline $8 a$ & $2.8 \pm 4.2$ & $1.2 \pm 4.1$ & $-1.5 \pm 3.8$ & $2.1 \pm 4.4$ & $-0.6 \pm 3.9$ & $-1.1 \pm 4.2$ & $0.8 \pm 4.0$ & $-2.7 \pm 3.7$ & $-0.6 \pm 3.8$ & $-1.6 \pm 3.8$ \\
\hline $9 a$ & $2.0 \pm 4.2$ & $-1.7 \pm 4.1$ & $0.0 \pm 3.7$ & $-1.5 \pm 4.4$ & $-0.3 \pm 3.9$ & $-1.8 \pm 4.1$ & $-2.0 \pm 4.0$ & $-0.1 \pm 3.7$ & $-0.4 \pm 3.9$ & $-0.1 \pm 3.8$ \\
\hline $10 \mathrm{a}$ & $2.7 \pm 4.8$ & $-2.3 \pm 4.5$ & $-5.1 \pm 4.3$ & $5.4 \pm 5.2$ & $0.5 \pm 5.3$ & $5.8 \pm 5.1$ & $4.1 \pm 4.5$ & $0.3 \pm 4.6$ & $6.2 \pm 4.3$ & $-1.5 \pm 4.7$ \\
\hline $11 \mathrm{a}$ & $0.6 \pm 4.4$ & $-2.2 \pm 4.0$ & $-2.9 \pm 3.9$ & $4.5 \pm 4.4$ & $4.0 \pm 4.0$ & $4.8 \pm 4.1$ & $0.8 \pm 4.1$ & $0.3 \pm 3.8$ & $3.2 \pm 3.8$ & $-3.8 \pm 3.9$ \\
\hline $12 \mathrm{a}$ & $-3.5 \pm 4.2$ & $-6.1 \pm 4.1$ & $-2.6 \pm 3.7$ & $2.0 \pm 4.4$ & $5.4 \pm 3.9$ & $5.9 \pm 4.1$ & $-2.6 \pm 4.0$ & $0.8 \pm 3.9$ & $3.5 \pm 3.8$ & $-4.4 \pm 3.9$ \\
\hline $13 a$ & $2.1 \pm 4.2$ & $-1.6 \pm 4.1$ & $-3.8 \pm 3.9$ & $-1.0 \pm 4.5$ & $-3.0 \pm 4.1$ & $-1.0 \pm 4.2$ & $-1.2 \pm 4.1$ & $-3.4 \pm 3.9$ & $0.7 \pm 3.7$ & $-0.2 \pm 4.0$ \\
\hline $14 \mathrm{a}$ & $3.1 \pm 4.2$ & $1.9 \pm 4.0$ & $-1.5 \pm 3.7$ & $1.3 \pm 4.4$ & $-1.6 \pm 3.9$ & $-2.5 \pm 4.1$ & $-2.0 \pm 4.0$ & $-4.5 \pm 3.7$ & $-3.4 \pm 3.7$ & $-3.0 \pm 4.1$ \\
\hline $15 \mathrm{a}$ & $-2.0 \pm 4.9$ & $-9.8 \pm 4.7$ & $-6.0 \pm 4.3$ & $13.5 \pm 6.5$ & $18.6 \pm 6.3$ & $21.7 \pm 7.4$ & $3.9 \pm 4.5$ & $7.7 \pm 4.7$ & $12.3 \pm 4.3$ & $-10.8 \pm 7.5$ \\
\hline $16 \mathrm{a}$ & $-0.6 \pm 4.2$ & $-3.3 \pm 4.1$ & $-2.6 \pm 3.8$ & $-1.0 \pm 4.5$ & $-0.1 \pm 4.0$ & $0.5 \pm 4.1$ & $-0.3 \pm 4.0$ & $0.6 \pm 3.8$ & $3.1 \pm 3.8$ & $1.1 \pm 3.8$ \\
\hline $17 \mathrm{a}$ & $5.3 \pm 4.9$ & $-3.5 \pm 4.5$ & $-6.7 \pm 4.4$ & $-11.3 \pm 5.3$ & $-12.3 \pm 5.0$ & $-9.9 \pm 5.0$ & $-1.8 \pm 4.9$ & $-5.4 \pm 4.5$ & $0.3 \pm 5.0$ & $7.5 \pm 4.9$ \\
\hline $18 \mathrm{a}$ & $-0.6 \pm 5.8$ & $-7.3 \pm 4.8$ & $-8.5 \pm 4.7$ & $-4.6 \pm 5.2$ & $-4.9 \pm 5.9$ & $-3.4 \pm 5.5$ & $-3.6 \pm 5.5$ & $-7.4 \pm 4.3$ & $2.3 \pm 4.5$ & $5.5 \pm 5.1$ \\
\hline $19 \mathrm{a}$ & $-4.3 \pm 4.8$ & $-6.4 \pm 4.5$ & $-1.6 \pm 4.3$ & $-7.2 \pm 6.2$ & $-5.2 \pm 5.1$ & $-3.5 \pm 5.1$ & $5.1 \pm 4.6$ & $9.9 \pm 4.5$ & $11.1 \pm 4.2$ & $14.1 \pm 4.9$ \\
\hline star & $12 \mathrm{~b}$ vs. $12 \mathrm{a}$ & 13 vs. $12 \mathrm{a}$ & 13 vs. $12 b$ & 14 vs. $12 \mathrm{a}$ & 14 vs. $12 b$ & 14 vs. 13 & 15 vs. $12 \mathrm{a}$ & 15 vs. $12 b$ & 15 vs. 13 & 15 vs. 14 \\
\hline $1 b$ & $3.5 \pm 2.4$ & -2.2 & -12.2 & $0.9 \pm 3.8$ & $-3.3 \pm 3.7$ & 15.8 & $-0.5 \pm 3.9$ & $-4.2 \pm 3.7$ & 9.0 & $-1.6 \pm 3.4$ \\
\hline $2 b$ & $-0.8 \pm 2.4$ & -2.6 & -7.3 & $-1.6 \pm 3.7$ & $-0.5 \pm 3.7$ & 13.6 & $0.4 \pm 3.8$ & $1.8 \pm 3.6$ & 10.3 & $1.9 \pm 3.7$ \\
\hline $3 b$ & $-1.7 \pm 3.4$ & -6.0 & -10.4 & $-6.5 \pm 4.5$ & $-4.9 \pm 4.3$ & 12.0 & $-1.0 \pm 4.2$ & $0.2 \pm 4.3$ & 11.4 & $5.6 \pm 4.3$ \\
\hline $4 b$ & $-0.7 \pm 3.2$ & 0.0 & -5.1 & $-4.4 \pm 4.3$ & $-4.2 \pm 4.4$ & 7.3 & $-0.1 \pm 4.2$ & $0.0 \pm 4.2$ & 7.9 & $4.6 \pm 4.0$ \\
\hline $5 b$ & $1.2 \pm 2.4$ & 0.5 & -6.3 & $-4.7 \pm 3.8$ & $-5.7 \pm 3.7$ & 7.0 & $0.4 \pm 3.8$ & $-0.7 \pm 3.7$ & 6.8 & $5.1 \pm 3.6$ \\
\hline $6 \mathrm{~b}$ & $-1.8 \pm 3.5$ & -0.8 & -4.6 & $-2.7 \pm 4.4$ & $-1.8 \pm 4.8$ & 8.8 & $1.3 \pm 4.5$ & $3.3 \pm 4.1$ & 9.5 & $4.3 \pm 5.0$ \\
\hline $7 \mathrm{~b}$ & $-7.3 \pm 3.7$ & -12.7 & -11.2 & $-7.8 \pm 4.7$ & $0.4 \pm 4.5$ & 14.0 & $-4.8 \pm 4.4$ & $0.8 \pm 4.5$ & 16.0 & $2.3 \pm 4.5$ \\
\hline $8 b$ & $-1.4 \pm 3.2$ & 0.4 & -3.8 & $3.8 \pm 4.7$ & $4.8 \pm 4.5$ & 14.3 & $-3.2 \pm 4.3$ & $-1.8 \pm 4.2$ & 4.2 & $-4.7 \pm 3.9$ \\
\hline $9 b$ & $-2.7 \pm 2.5$ & -3.3 & -6.3 & $-11.0 \pm 3.9$ & $-8.6 \pm 3.7$ & 4.0 & $-8.4 \pm 3.6$ & $-5.8 \pm 3.7$ & 2.3 & $2.2 \pm 3.4$ \\
\hline $10 \mathrm{~b}$ & $-2.1 \pm 3.2$ & 2.9 & 0.2 & $-1.5 \pm 4.6$ & $1.5 \pm 4.5$ & 6.8 & $-3.8 \pm 4.2$ & $-0.9 \pm 4.4$ & 2.1 & $-1.7 \pm 4.6$ \\
\hline $11 b$ & $-1.2 \pm 2.4$ & 1.3 & -3.0 & $1.5 \pm 3.9$ & $2.8 \pm 4.0$ & 11.6 & $2.9 \pm 3.6$ & $4.0 \pm 3.6$ & 9.3 & $1.4 \pm 3.8$ \\
\hline $12 b$ & $-1.1 \pm 3.5$ & 0.7 & -4.7 & $-0.5 \pm 4.6$ & $-0.4 \pm 4.5$ & 9.7 & $1.1 \pm 4.9$ & $3.9 \pm 4.9$ & 10.9 & $2.5 \pm 4.7$ \\
\hline $13 b$ & $1.1 \pm 3.8$ & 3.6 & -2.6 & $3.2 \pm 5.1$ & $1.3 \pm 5.3$ & 9.4 & $-3.8 \pm 4.5$ & $-5.4 \pm 4.5$ & -0.4 & $-7.4 \pm 5.4$ \\
\hline \multirow[t]{19}{*}{$14 b$} & $0.9 \pm 3.2$ & 6.8 & 0.9 & $-1.0 \pm 4.3$ & $-2.2 \pm 4.5$ & 3.3 & $3.0 \pm 4.2$ & $2.1 \pm 4.1$ & 3.6 & $2.8 \pm 4.1$ \\
\hline & & Star & $14 \mathrm{a}$ vs. 13 & $14 \mathrm{~b}$ vs. 13 & $14 \mathrm{~b}$ vs. $14 \mathrm{a}$ & 15 vs. 13 & 15 vs. $14 \mathrm{a}$ & 15 vs. $14 b$ & & \\
\hline & & $1 \mathrm{c}$ & $-0.3 \pm 3.7$ & $-2.3 \pm 3.8$ & $-2.0 \pm 3.7$ & $-0.9 \pm 3.8$ & $-0.6 \pm 4.0$ & $1.4 \pm 3.4$ & & \\
\hline & & $2 \mathrm{c}$ & $0.1 \pm 3.7$ & $-1.9 \pm 4.0$ & $-1.7 \pm 3.7$ & $-4.3 \pm 3.7$ & $-4.2 \pm 3.7$ & $-2.2 \pm 3.5$ & & \\
\hline & & $3 c$ & $-1.3 \pm 3.8$ & $-2.0 \pm 3.9$ & $-0.4 \pm 3.6$ & $-2.1 \pm 3.8$ & $-0.8 \pm 3.8$ & $-0.1 \pm 3.5$ & & \\
\hline & & $4 c$ & $1.4 \pm 3.8$ & $-0.2 \pm 4.0$ & $-1.5 \pm 3.6$ & $-0.1 \pm 3.8$ & $-1.6 \pm 3.9$ & $0.0 \pm 3.4$ & & \\
\hline & & $5 c$ & $2.5 \pm 3.7$ & $4.8 \pm 3.7$ & $1.7 \pm 3.6$ & $3.2 \pm 4.0$ & $0.3 \pm 3.7$ & $-2.0 \pm 3.4$ & & \\
\hline & & $6 c$ & $1.4 \pm 4.0$ & $0.4 \pm 4.2$ & $-0.3 \pm 3.6$ & $0.3 \pm 3.8$ & $-0.9 \pm 3.7$ & $-0.6 \pm 3.4$ & & \\
\hline & & $7 \mathrm{c}$ & $3.4 \pm 3.9$ & $-1.4 \pm 3.8$ & $-4.9 \pm 3.6$ & $-4.3 \pm 3.8$ & $-7.5 \pm 3.7$ & $-2.8 \pm 3.7$ & & \\
\hline & & $8 \mathrm{c}$ & $-1.9 \pm 4.5$ & $3.9 \pm 4.9$ & $5.9 \pm 4.5$ & $6.2 \pm 4.5$ & $6.8 \pm 4.5$ & $-0.8 \pm 4.5$ & & \\
\hline & & $9 \mathrm{c}$ & $5.4 \pm 3.8$ & $1.8 \pm 3.7$ & $-3.6 \pm 3.7$ & $3.2 \pm 3.7$ & $-2.1 \pm 3.7$ & $1.4 \pm 3.5$ & & \\
\hline & & $10 \mathrm{c}$ & $4.2 \pm 4.7$ & $0.5 \pm 5.0$ & $-2.3 \pm 4.5$ & $-3.9 \pm 4.5$ & $-5.5 \pm 4.5$ & $-4.5 \pm 4.2$ & & \\
\hline & & $11 \mathrm{c}$ & $-0.7 \pm 3.8$ & $0.4 \pm 3.7$ & $1.1 \pm 3.6$ & $3.1 \pm 3.8$ & $3.8 \pm 3.7$ & $2.9 \pm 3.3$ & & \\
\hline & & $12 \mathrm{c}$ & $-1.4 \pm 4.5$ & $-7.0 \pm 4.5$ & $-4.4 \pm 4.5$ & $-4.0 \pm 4.7$ & $-0.8 \pm 4.5$ & $3.2 \pm 4.5$ & & \\
\hline & & $13 \mathrm{c}$ & $-1.4 \pm 3.8$ & $0.4 \pm 3.7$ & $1.9 \pm 3.7$ & $0.6 \pm 3.7$ & $1.6 \pm 3.7$ & $0.3 \pm 3.3$ & & \\
\hline & & $15 \mathrm{c}$ & $2.7 \pm 3.9$ & $2.0 \pm 3.7$ & $-0.8 \pm 3.7$ & $1.1 \pm 3.7$ & $-1.6 \pm 4.0$ & $-0.9 \pm 3.4$ & & \\
\hline & & $16 \mathrm{c}$ & $10.7 \pm 4.4$ & $3.5 \pm 4.6$ & $-5.1 \pm 4.5$ & $5.0 \pm 4.5$ & $-3.9 \pm 4.4$ & $3.2 \pm 4.2$ & & \\
\hline & & $17 \mathrm{c}$ & $-0.2 \pm 3.7$ & $0.5 \pm 3.7$ & $0.6 \pm 3.6$ & $-1.3 \pm 3.8$ & $-0.9 \pm 3.7$ & $-1.6 \pm 3.3$ & & \\
\hline & & $18 \mathrm{c}$ & $2.6 \pm 3.7$ & $4.1 \pm 3.7$ & $1.5 \pm 3.6$ & $1.2 \pm 3.8$ & $-1.9 \pm 3.8$ & $-3.4 \pm 3.4$ & & \\
\hline
\end{tabular}




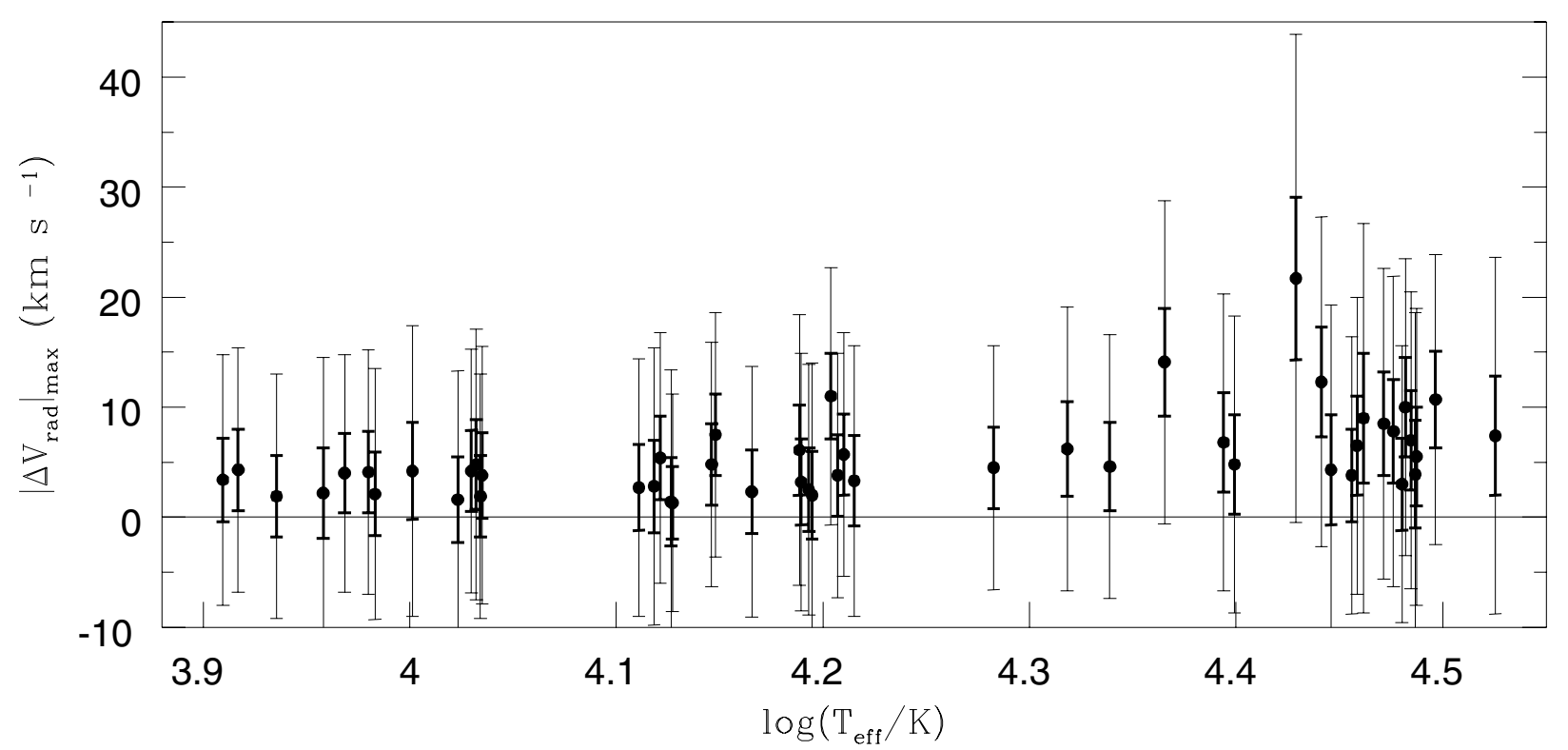

Fig. 9. Maximum RV variation for each star (in absolute value) measured in $\mathrm{H}_{\beta}$ wavelength, plotted as a function of $T_{\text {eff }}$. The thick errorbars are the $1 \sigma$ errors, while the thinner bars indicate the $3 \sigma$. There is no RV variation exceeding the $3 \sigma$ error bars.

We performed similar calculations also assuming a companion of $0.1 M_{\odot}$. This kind of system is a minority among the binary population in the field, but they do exist, as for example the well-known HW Vir system (Menzies \& Marang 1986). As shown in Fig. 11, the detection probability for such systems is very low in our survey, with the exception of the shortest (1-2 days) period binaries.

\subsection{Binary fraction estimate}

If the fraction of binaries in the sample is $f$ the probability of detecting $N_{\mathrm{B}}$ binaries out of a sample of $N$ stars is:

$P=\frac{N !}{\left(N-N_{\mathrm{B}}\right) ! N_{\mathrm{B}} !}(d f)^{N_{\mathrm{B}}}(1-\bar{d} f)^{N-N_{\mathrm{B}}}$

where $\bar{d}$ is the probability of detection weighted for the period distribution of binaries. In our survey $N_{\mathrm{B}}=0$ and $N=18$, therfore,

$P=(1-\bar{d} f)^{18}$.

Since we found no binaries, the probability $P$ has a unitary maximum in $f=0$. Then, for increasing values of $f$, it falls rapidly to zero. The exact shape of the function depends on the assumed period distribution, which is very uncertain. We performed the calculations for two limiting cases, a flat distribution and a truncated Gaussian centered on $P=1$ day and with $\log (P /$ days $)=1$ as FWHM, as also assumed by Maxted et al. (2001) and Napiwotzki et al. (2004). This distribution seems to follow the observations of field sdB stars, although the known data are still too few and this is just a first guess. The results on $P$ obtained with the two distributions are extremely similar to within $1 \%$, because the decline of the Gaussian wings in the second case compensates for higher (lower) values of the sensitivity for short (long) periods, and the weighted mean is very similar. The value of $P$ reaches 0.05 for $f=0.20$, in both cases. Then we can conclude that the binary fraction among EHB stars in NGC 6752 is lower then $20 \%$ at a confidence level of $95 \%$.

\section{Discussion and conclusions}

The results of Fig. 9 clearly indicate that there is no close binary system in our sample. The RV variations observed during the four nights, with different temporal samplings, are all within the estimated errors. These errors $\left(\approx 4-6 \mathrm{~km} \mathrm{~s}^{-1}\right)$ are significantly smaller than the radial velocity variations we were expecting based on the results for field sdB stars.

In the compilation by Morales-Rueda et al. (2003), for all the 38 field sdBs with known orbital periods, the RV semiamplitude is always greater than $30 \mathrm{~km} \mathrm{~s}^{-1}$. These binaries should have been easily detected in our survey at a 5-6 sigma level. If the binary fraction of the EHB stars in NGC 6752 is the same as among the field sdB stars listed in Maxted et al. (2001), we would expect that $13 \pm 2$ of the 18 stars with $T_{\mathrm{e}}>22000 \mathrm{~K}$ in our sample should be close binaries; given the period distribution of Morales-Rueda et al. (2003), 80\% of them should have a period $P<5$ days and, therefore, be easily detected with our temporal sampling. Indeed, as shown in the previous section, our survey should be able to detect $85 \%$ of close binaries with $P<5$ days of the kind found among the field sdB stars. I.e., if the binary fraction among the hot cluster EHB stars were the same as among field sdB stars, we would have expected to find 9 binaries with $\mathrm{RV}$ variations $>20 \mathrm{~km} \mathrm{~s}^{-1}$.

We found no significant RV variation at 3 sigma level $\left(15-18 \mathrm{~km} \mathrm{~s}^{-1}\right)$. Only 18 measurements showed a variation exceeding 2 sigmas $\left(10-12 \mathrm{~km} \mathrm{~s}^{-1}\right)$. However, assuming a Gaussian distribution for our errors, we would expect that 19.6 measures among our $432 \mathrm{RV}$ difference estimates should exceed the 2 sigma level; therefore, these variations too cannot be considered significant. As demonstrated in Sect. 5.4, 

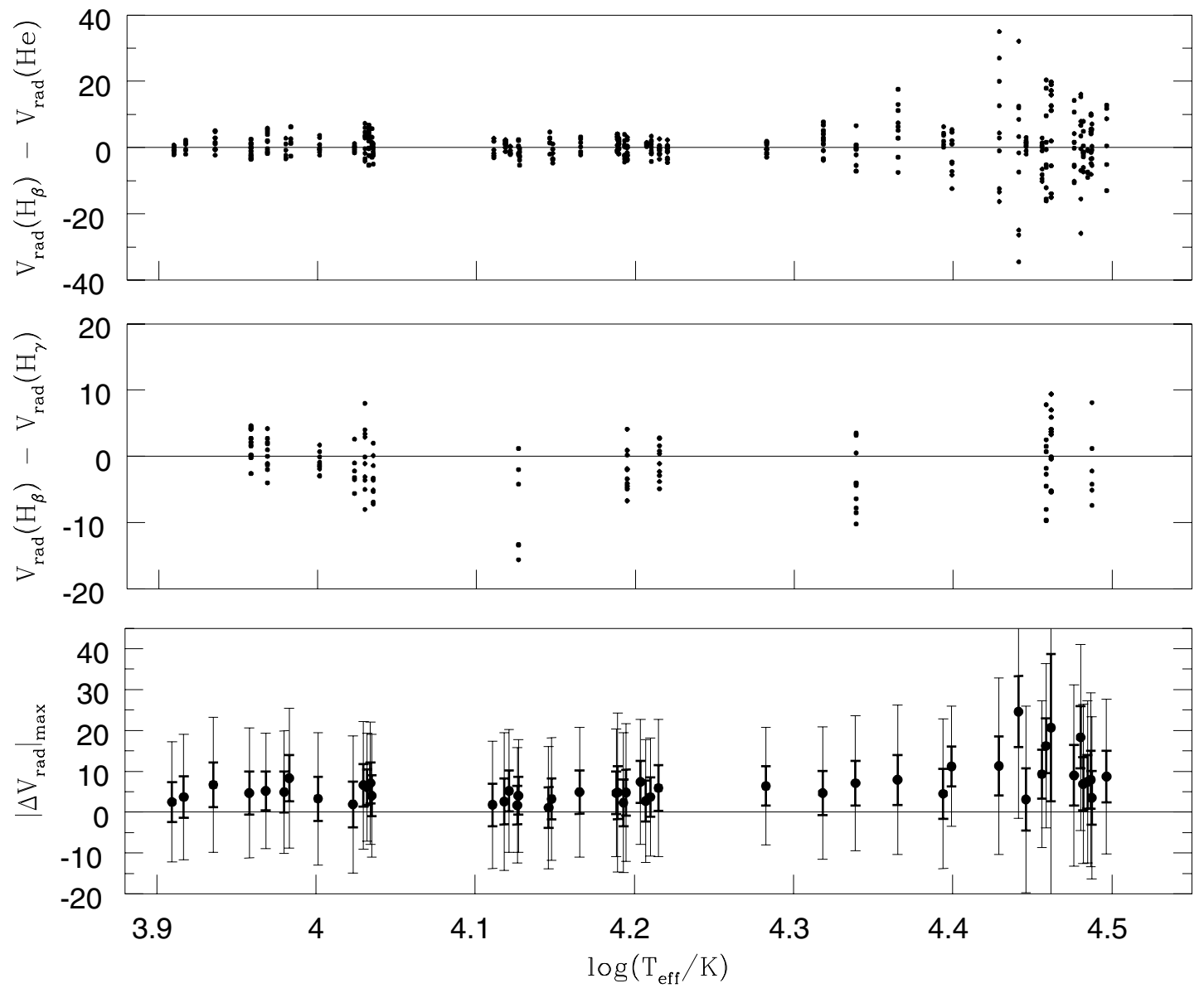

Fig. 10. Summary of the results in other wavelengths, plotted vs. effective temperature of the stars. Top panel: differences between the results in $\mathrm{H}_{\beta}$ and the weak lines. Central panel: differences between the results in $\mathrm{H}_{\beta}$ and $\mathrm{H}_{\gamma}$. Bottom panel: maxima $\mathrm{RV}$ variations measured with weak lines. The thick error bar indicates the $1 \sigma$ interval, and the thin one is the $3 \sigma$.

these results imply that the fraction of close binaries among EHB stars in NGC 6752 is $<20 \%$, at a $95 \%$ confidence level. This result is in sharp contrast with that of Peterson et al. (2002), who concluded, from the measurement of radial velocity variations of $30 \mathrm{EHB}$ stars in NGC 6752, that the majority of them are close binaries. Peterson et al. (2002) unfortunately have not published more details about their investigation so far, and therefore, it remains to be established whether the different conclusions are due to the lower $\mathrm{S} / \mathrm{N}$ of Peterson et al. (2002) data (who used the multifiber Hydra spectrograph mounted on a $4 \mathrm{~m}$ size telescope) or to some physical reasons. Interestingly enough, apparently, our sample of stars is different from their sample. In particular, according to Peterson (priv. comm.), their EHB stars are on average located in a less crowded, more external environment than ours (though our stars are evenly distributed from $\sim 100^{\prime \prime}$ to $\sim 500^{\prime \prime}$ from the cluster center, i.e. out to about 3.5 half-mass radii, see Fig. 2). We have only five stars in common, of which only two are EHB stars and for which also Peterson et al. (2002) do not find evidence of radial velocity variations (Peterson, priv. comm.).

In summary, we are forced to conclude that most $(>80 \%)$ of the hot EHB stars in NGC 6752 are not close binaries. This result poses a number of problems. First of all, it implies that the mechanism for the formation of EHB stars in GCs does not

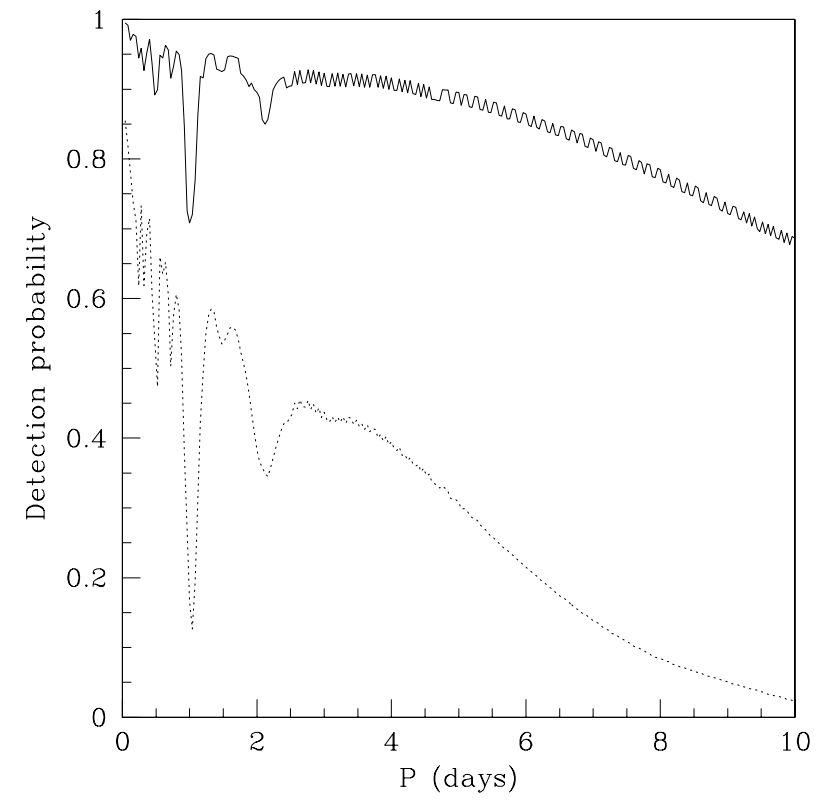

Fig. 11. Probability of detecting a binary system in our observations as a function of its period $P$. The solid line indicates the probability calculated for an sdB star and a companion of $0.5 M_{\odot}$, the dotted line for a companion of $0.1 M_{\odot}$, assuming an EHB star of $0.5 M_{\odot}$ in both cases. 
involve a large envelope mass loss enhanced by tidal interaction within a close binary at variance with what happens for the field sdBs. Or, at least, this cannot be the dominant formation mechanism. In a sense, this result is not completely unexpected. The typical companions of field sdB stars are relatively massive stars $\left(0.3-0.5 M_{\odot}\right.$, see Maxted et al. 2004). If the same binary systems are the source of the EHB stars in GCs, they should originally have had a mass that is on average larger $\left(\geq 1 M_{\odot}\right)$ than the mass of a single star at the TO $\left(\sim 0.8 M_{\odot}\right)$ and, therefore, should be more concentrated than the upper MS/RGB stars because of mass segregation. Despite a number of attempts, there is no compelling evidence at the moment that EHB stars are, on average, more concentrated than cooler HB stars in the same clusters, as we would expect if they were members of a binary system with similar properties to the binaries producing field sdBs, and as we find, e.g., for the blue stragglers in all the observed GCs (Piotto et al. 2004). Instead, recent surveys (e.g. by Bedin et al. 2000, in NGC 2808, a prototype EHB cluster) have shown that no clear radial gradient of the EHB stars can be detected. This empirical evidence, coupled with the results of the present paper, implies that: either 1) the companions of the EHB stars have very low masses (so that the sum of the main sequence mass of the EHB stars and that of the companion do not differ much from the mass of a typical MS star); or 2) cluster EHB stars all have periods much longer than 5 days; or 3 ) cluster EHB stars are not binaries.

In any case, we are forced to conclude that in GCs there are different formation mechanisms of EHB stars with respect to the main formation channels of field sdBs. It is possible that the dynamical evolution of GCs have removed the primordial binaries able to produce sdB stars, at least in the inner part of the cluster. This is an interesting possibility. Indeed, Piotto et al. (2004) have shown that the frequency of blue stragglers (BSs) in the cores of GCs is significantly smaller than the frequency of field BSs, and that there is an anticorrelation between the frequency of BSs and the GC total mass. Davies et al. (2004) suggest that this is likely due to the fact that in more massive clusters, where the encounter probability is higher, binary evolution is accelerated by similar mechanisms to those proposed by Davies \& Hansen (1998) to explain the formation of pulsars in GCs. In this way, the frequency of BSs (from primordial binaries) could have been much higher in the past, but now there are much fewer binaries to produce them via the merger channel. However, the GC environment can favor different formation mechanisms for the EHB stars. Again, something similar happens for the BSs. Piotto et al. (2004) show that the luminosity functions of the BSs in the core of massive clusters differ significantly from those in less massive ones, and the former is compatible with BSs mainly formed via collision of stars (more probable in more massive clusters).

Interestingly enough, Recio-Blanco et al. (2005) show that more massive clusters also show more extended HBs. If the Peterson et al. (2002) results are confirmed by a more extended survey. i.e. if at least a fraction of the EHB stars in the outskirts of the clusters are indeed close binaries (at variance with what we find in the more internal regions), we will have further evidence of the effect of the environment on the formation of EHB (or sdB) stars. This would also explain the sharp difference between the frequency of close binaries among cluster EHB stars and field sdBs. The results of the present paper and of Peterson et al. (2002) might suggest that two different channels of production of EHB stars are at work, at least in some GCs. Again, it is tempting to recall that there is compelling evidence of two channels of BS production in the same GC. It has been shown that the radial distribution of BSs in M 3 (Ferraro et al. 1997) and in 47Tuc (Ferraro et al. 2004) are clearly bimodal and that the BS properties in the inner regions and in the outskirts of this cluster are also different. Mapelli et al. (2004) show that the radial distribution of BS in 47Tuc supports the hypothesis that the BSs produced in the center are caused mainly by collisions, while the BS in the cluster outskirts (for $r>30$ core radii, i.e. for distances from the center greater than 4 half mass radii) must all result from the merge of primordial binaries, which survived because of the much slower dynamical evolution in the external part of the cluster. A similar scenario could also be at work for EHB stars, with EHB stars in the external part of the cluster coming from the close binary-evolution channel suggested for the production of field sdB stars. It would be interesting to use the appropriate dynamical and stellar evolution models to investigate the possibility that EHB stars in GCs can be formed preferentially by stellar collisions in their more central parts. Surely, our results and those of Peterson et al. (2002) call for a much more extended observing campaign to search for close binaries among EHB stars in this and other GCs.

Another interesting possibility that needs to be (observationally) explored is that cluster EHB stars are produced in binaries with significantly longer periods or with significantly less massive companion than in field sdBs. As shown in Fig. 11, our survey could find only binaries with relatively large-mass companions, and as it only has a 4-day temporal coverage, it is totally insensitive to those binaries with periods longer than 10 days. We note that the Han et al. (2003) models for the production of $\mathrm{sdB}$ stars predict a large number of long period ( $\sim 100$ days) binaries. A follow-up observing campaign is needed to verify if there are binaries like these among cluster EHB stars; in any case, long-period binaries seem to be a minority among field sdBs, so that the question to why field and cluster EHB stars should form through different mechanisms remains unanswered.

Of course, it is equally possible that dynamical evolution and binaries have nothing to do with the formation of EHB stars in GCs. Unlike field sdBs, which can have progenitors with rather different masses, all the cluster EHB stars start their evolution from a well defined TO mass $\left(\sim 0.8 M_{\odot}\right.$, slightly depending on cluster age and metallicity), because of the very small (compatible with zero) age and metallicity dispersion among stars in a given GC. There must be an extremely well-tuned (unlikely?) mass-loss mechanism to produce a star with the very small core and envelope mass dispersion of the cluster EHB stars. Other explanations are possible. As discussed in the introduction, recent results on very massive clusters like $\omega$ Centauri (Piotto et al. 2005) and NGC 2808 (D'Antona et al. 2005) seem to indicate that the EHBs in GCs could represent the evolved population of a second generation of stars formed by material enriched in He because of the pollution by the 
ejecta of SNe and/or intermediate mass AGBs from a first generation of stars. In this hypothesis, it is possible that more massive clusters are better able to keep part of the ejecta, explaining the correlation found by Recio-Blanco et al. (2005). One should keep in mind, however, that not only the total mass, but also the concentration of a globular cluster determine the depth of its gravitational potential and thereby its ability to retain enriched material.

There is an interesting point to add before concluding this discussion. As noted in the introductory section, Napiwotzki et al. (2004) found a much lower (42\%) binary frequency among field sdBs than did Maxted et al. (2001). On one hand, even with this low binary frequency, we would expect to find 5 binaries among our sampled 18 EHB stars in NGC 6752. Napiwotzki et al. (2004) suggest that the difference in mean apparent magnitude between their sample and the one of Maxted et al. (2001) may imply a difference in the populations sampled by the two surveys. Although this has not yet been proven, attributing the observed difference in binary fraction to a population difference would mean that binaries are substantially less frequent among thick disk-halo sdBs, and it would imply a trend with age and/or metallicity. On average, our cluster EHB stars are expected to be older, and possibly more metal poor than the Napiwotzki et al. sample, and, apparently, the fraction of binaries among them is even smaller than among the Napiwotzki et al. sample. It is currently unclear if these differences are related to the different environments, ages, or metallicities, or to a combination of these parameters. Supporting evidence for the possible influence of abundance differences comes from the observed abundance anomalies in GC red giants (Catelan 2005; Gratton et al. 2004, and references therein), which are not seen in field red giants (Gratton et al. 2000). As suggested by many in the literature (e.g. Vandenberg \& Smith 1988; Sweigart 1997; D'Antona et al. 2002), these abundance anomalies (either primordial, or due to mixing effects) can affect the RGB evolution and, consequently, have an impact on the properties of HB stars including temperatures, luminosities, gravities, and pulsation characteristics.

Acknowledgements. G.P. acknowledges the support by MIUR within the PRIN2003 program. R.A.M. acknowledges support from the Chilean Centro de Astrofísica FONDAP (No. 15010003). We thank Ron Webbink for useful discussions and for pointing out the problem of the small core and envelope mass dispersion of EHB stars when trying to interpret their origin in terms of mass loss within a binary system. A.R.B. acknowledges the support of the European Space Agency. We thank the staff at Paranal observatory for their support during the observations.

\section{References}

Bailyn, C. D. 1993, in Structure and Dynamics of Globular Clusters, ASP Conf. Ser., 50, 191

Bedin, L. R., Piotto, G., Zoccali, M., et al. 2000, A\&A, 363, 159

Bedin, L. R., Piotto, G., Anderson, J., et al. 2004, ApJ, 605, L125

Behr, B. B. 2003, ApJS, 149, 67

Brault, J. W., \& White, O. R. 1971, A\&A, 13, 169
Brown, T. M., Bowers, C. W., Kimble, R. A., \& Ferguson, H. C. 2000, ApJ, 529, L89

Busso, G., Moehler, S., Zoccali, M., Heber, U., \& Yi, S. K. 2005, ApJ, 633, L29

Caloi, V., \& D'Antona, F. 2005, A\&A, 435, 987

Catelan, M. 2005, in Resolved Stellar Populations, ASP Conf. Ser., in press [arXiv: astro-ph/0507464]

Code, A. D. 1969, PASP, 81, 475

D’Antona, F., Caloi, V., Montalbán, J., Ventura, P., \& Gratton, R. 2002, A\&A, 395, 69

D’Antona, F., Bellazzini, M., Caloi, V., et al. 2005, ApJ, in press [arXiv: astro-ph/0505347]

Davies, M. B., \& Hansen, B. M. S. 1998, MNRAS, 301, 15

Davies, M. B., Piotto, G., \& de Angeli, F. 2004, MNRAS, 349, 129

D’Cruz, N. L., Dorman, B., Rood, R. T., \& O'Connell, R. W. 1996, ApJ, 466, 359

Dubath, P., Meylan, G., Mayor, M., \& Magain, P. 1990, A\&A, 239, 142

Faulkner, J. 1966, ApJ, 144, 978

Faulkner, J. 1972, ApJ, 173, 401

Ferraro, F. R., Paltrinieri, B., Fusi Pecci, F., et al. 1997, A\&A, 324, 915

Ferraro, F. R., Paltrinieri, B., Pecci, F. F., Rood, R. T., \& Dorman, B. 1998, ApJ, 500, 311

Ferraro, F. R., Beccari, G., Rood, R. T., et al. 2004, ApJ, 603, 127

Fusi Pecci, F., Ferraro, F. R., Bellazzini, M., et al. 1993, AJ, 105, 1145

Glaspey, J. W., Michaud, G., Moffat, A. F. J., \& Demers, S. 1989, ApJ, 339,926

Gratton, R. G., Sneden, C., Carretta, E., \& Bragaglia, A. 2000, A\&A, 354,169

Gratton, R., Sneden, C., \& Carretta, E. 2004, ARA\&A, 42, 385

Greggio, L., \& Renzini, A. 1990, ApJ, 364, 35

Greggio, L., \& Renzini, A. 1999, Mem. Soc. Astron. Ital., 70, 691

Han, Z., Podsiadlowski, P., Maxted, P. F. L., Marsh, T. R., \& Ivanova, N. 2002, MNRAS, 336, 449

Han, Z., Podsiadlowski, P., Maxted, P. F. L., \& Marsh, T. R. 2003, MNRAS, 341, 669

Harris, W. E. 1996, AJ, 112, 1487

Heber, U., Moehler, S., Napiwotzki, R., Thejll, P., \& Green, E. M. 2002, A\&A, 383, 938

Heber, U., Edelmann, H., Lisker, T., \& Napiwotzki, R. 2003, A\&A, 411, L477

Heggie, D. C. 1975, MNRAS, 173, 729

Horne, K. 1986, PASP, 98, 609

Hoyle, F., \& Schwarzschild, M. 1955, ApJS, 2, 1

Hut, P., McMillan, S., Goodman, J., et al. 1992, PASP, 104, 981

Iben, I. J. 1990, ApJ, 353, 215

Iben, I. J., \& Rood, R. T. 1970, ApJ, 161, 587

Lee, Y., Joo, S., Han, S., et al. 2005, ApJ, 621, L57

Lisker, T., Heber, U., Napiwotzki, R., et al. 2005, A\&A, 430, 223

Mapelli, M., Sigurdsson, S., Colpi, M., et al. 2004, ApJ, 605, L29

Maxted, P. F. L., Heber, U., Marsh, T. R., \& North, R. C. 2001, MNRAS, 326, 1391

Maxted, P. F. L., Morales-Rueda, L., \& Marsh, T. R. 2004, Ap\&SS, 291, 307

Mengel, J. G., Norris, J., \& Gross, P. G. 1976, ApJ, 204, 488

Menzies, J. W., \& Marang, F. 1986, in Instrumentation and Research Programmes for Small Telescopes, IAU Symp., 118, 305

Moehler, S., Sweigart, A. V., Landsman, W. B., \& Heber, U. 2000, A\&A, 360, 120

Momany, Y., Piotto, G., Recio-Blanco, A., et al. 2002, ApJ, 576, L65

Morales-Rueda, L., Maxted, P. F. L., Marsh, T. R., North, R. C., \& Heber, U. 2003, MNRAS, 338, 752 
Napiwotzki, R., Karl, C. A., Lisker, T., et al. 2004, Ap\&SS, 291, 321

Peterson, R. C., Green, E. M., Rood, R. T., Crocker, D. A., \& Kraft, R. P. 2002, in Omega Centauri, A Unique Window into Astrophysics, ASP Conf. Ser., 265, 55

Piotto, G., Zoccali, M., King, I. R., et al. 1999, AJ, 118, 1727

Piotto, G., King, I. R., Djorgovski, S. G., et al. 2002, A\&A, 391, 945

Piotto, G., De Angeli, F., King, I. R., et al. 2004, ApJ, 604, L109

Piotto, G., Villanova, S., Bedin, L. R., et al. 2005, ApJ, 621, 777

Pooley, D., Lewin, W. H. G., Homer, L., et al. 2002, ApJ, 569, 405

Recio-Blanco, A., Piotto, G., Aparicio, A., \& Renzini, A. 2004, A\&A, 417, 597

Recio-Blanco, A., Aparicio, A., Piotto, G., de Angeli, F., \& Djorgowsky, S. G. 2005, A\&A, submitted

Rich, R. M., Sosin, C., Djorgovski, S. G., et al. 1997, ApJ, 484, L25

Rubenstein, E. P., \& Bailyn, C. D. 1997, ApJ, 474, 701
Sandage, A., \& Wallerstein, G. 1960, ApJ, 131, 598

Sandage, A., \& Wildey, R. 1967, ApJ, 150, 469

Soker, N., Catelan, M., Rood, R. T., \& Harpaz, A. 2001, ApJ, 563, L69

Sosin, C., Dorman, B., Djorgovski, S. G., et al. 1997, ApJ, 480, L35

Sweigart, A. V. 1997, ApJ, 474, L23

Tantalo, R., Chiosi, C., Bressan, A., \& Fagotto, F. 1996, A\&A, 311, 361

Tonry, J., \& Davis, M. 1979, AJ, 84, 1511

van den Bergh, S. 1967, AJ, 72, 70

Vandenberg, D. A., \& Smith, G. H. 1988, PASP, 100, 314

Vink, J. S., \& Cassisi, S. 2002, A\&A, 392, 553

Wilson, L. A., \& Bowen, G. H. 1984, Nature, 312, 429

Yong, H., Demarque, P., \& Yi, S. 2000, ApJ, 539, 928

Zucker, S., \& Mazeh, T. 1994, ApJ, 420, 806 